\title{
CROSS SECTIONS AND SOURCE SPECTRUM FOR NAGASAKI DOSE RECONSTRUCTION FOR RISK ESTIMATION
}

\author{
D. B. Simpson \\ R. T. Santoro \\ Y. Y. Azmy \\ J. V. Pace III
}





\title{
CROSS SECTIONS AND SOURCE SPECTRUM FOR NAGASAKI DOSE RECONSTRUCTION FOR RISK ESTIMATION
}

\author{
D. B. Simpson \\ R. T. Santoro \\ Y. Y. Azmy \\ J. V. Pace III
}

Computational Physics and Engineering

Date Published: May 2001

Prepared by the

OAK RIDGE NATIONAL LABORATORY

Oak Ridge, TN 37831-6363

Managed by

UT-BATTELLE, LLC

for the

U.S. DEPARTMENT OF ENERGY

under contract DE-AC05-00OR22725 



\section{CONTENTS}

ABSTRACT $\ldots \ldots \ldots \ldots \ldots \ldots \ldots \ldots \ldots \ldots \ldots \ldots \ldots \ldots \ldots \ldots \ldots \ldots \ldots \ldots \ldots \ldots \ldots$

1. INTRODUCTION $\ldots \ldots \ldots \ldots \ldots \ldots \ldots \ldots \ldots \ldots \ldots \ldots \ldots \ldots \ldots \ldots \ldots \ldots$. $\ldots \ldots \ldots$

2. SUMMARY DESCRIPTION OF RELATED CROSS-SECTION LIBRARIES ‘ . . . . 3

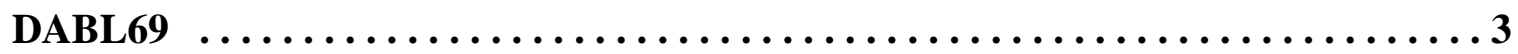

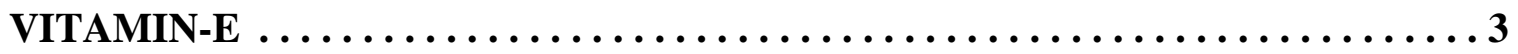

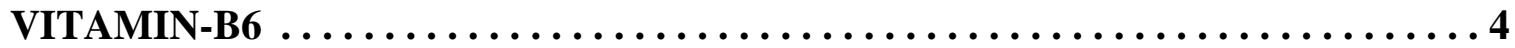

NEW LIBRARIES $\ldots \ldots \ldots \ldots \ldots \ldots \ldots \ldots \ldots \ldots \ldots \ldots \ldots \ldots \ldots \ldots$

3. DESCRIPTION OF NEUTRON AND PHOTON SOURCE SPECTRA $\ldots \ldots \ldots \ldots \ldots .7$

4. REFERENCES $\ldots \ldots \ldots \ldots \ldots \ldots \ldots \ldots \ldots \ldots \ldots \ldots \ldots \ldots \ldots \ldots \ldots \ldots \ldots \ldots$

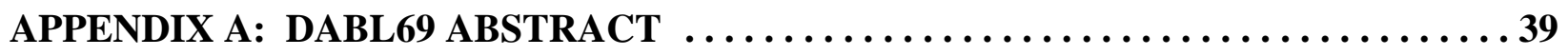

APPENDIX B: KAOS-LIB-V ABSTRACT $\ldots \ldots \ldots \ldots \ldots \ldots \ldots \ldots \ldots \ldots \ldots \ldots \ldots$

APPENDIX C: RESPONSE FUNCTIONS IN THE KAOS/LIB-V LIBRARY . . . . . . 47

APPENDIX D: VITAMIN-E ABSTRACT $\ldots \ldots \ldots \ldots \ldots \ldots \ldots \ldots \ldots \ldots \ldots \ldots \ldots$

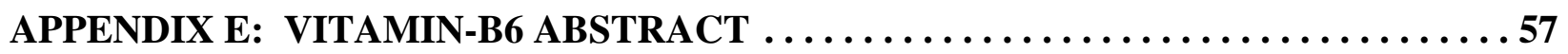





\section{LIST OF TABLES}

Table 1. Energy Boundaries for Neutron Groups $\ldots \ldots \ldots \ldots \ldots \ldots \ldots$

Table 2. Energy Boundaries for Photon Groups $\ldots \ldots \ldots \ldots \ldots \ldots \ldots \ldots \ldots$

Table 3. Materials in the DABL69 Library with Standard Weighting . . . . . . . 17

Table 4. Nuclides Available in VITAMIN-B6 . . . . . . . . . . . . . . . 18

Table 5. List of Materials in New Libraries. . . . . . . . . . . . . . . . 20



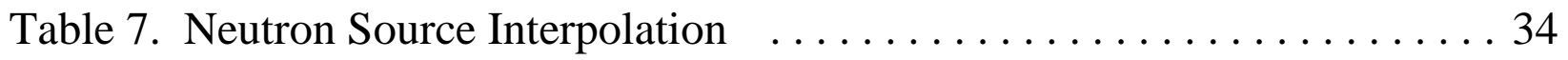

Table 8. Photon Source Interpolation . . . . . . . . . . . . . . . . . 35 



\section{LIST OF FIGURES}

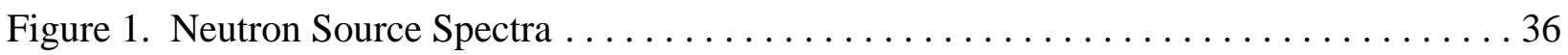

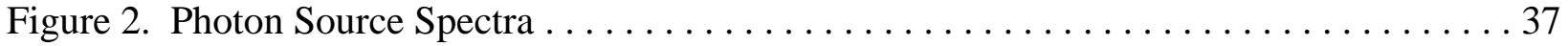





\begin{abstract}
A new broad-group cross-section library with the DABL69 energy group structure and based on ENDF/B-VI Release 3 data has been created. This new library was prepared using the standard weighting function in the VITAMIN-B6 fine-group cross-section library. The new broad-group library was prepared as a requisite for a project in which various factors in estimated doses to surviving Nagasaki factory workers are being investigated. This report documents the procedure used in creating the new library and provides specifications and limitations of the data which determine the usefulness of the library for problem-specific applications. The published neutron and photon source terms for the Nagasaki calculations have been interpolated to the energy group structure of the new library and are also presented within this report.
\end{abstract}





\section{INTRODUCTION}

A project at ORNL to investigate the impact of various factors on estimated doses to Nagasaki factory workers is ongoing. The newer dose estimates generated in this project will be verified by comparisons between calculated and measured chromosome aberration yields for the Nagasaki factory workers. Calculations for this project are to use cross sections from the latest ENDF/B-VI evaluation. The purpose of this report is to document the procedure that was used in creating a new broad-group cross-section library based on ENDF/B-VI, and to define the specifications needed to successfully use the library.

VITAMIN-B6 ${ }^{2}$ is a fine-group (199-neutron, 42-photon) library based on ENDF/B-VI Release 3. Because VITAMIN-B6 is distributed in AMPX ${ }^{3}$ master library format, an infinite dilution ANISN ${ }^{4}$ -format library was created as part of the task to prepare a new cross-section library. A broad-group library is also needed because of the large storage requirement for discrete ordinates calculations in two and three dimensions. Since the DABL69 $9^{5}$ broad-group (46-neutron, 23-photon) library was used in previous calculations to estimate the doses to Nagasaki factory workers, its group structure was chosen for the new broad-group library.

The VITAMIN-B6 library, rather than the ENDF/B-VI data files, was used to create the new broadgroup library having the DABL69 group structure. Creation of a multigroup library may involve considerable effort to process pointwise data into multigroup form. Fine-group libraries may generally be considered general purpose because the concern for application-dependent energy weighting has been lessened as a result of the fine-group structure. The dominant uncertainty with broad-group libraries is generally with regard to the energy weighting functions used in collapsing the fine-group data into broad-group data. For a selected set of problems, judicious selection of the energy group structure can reduce the sensitivity of the computed responses to the weighting functions. Since many of the VITAMIN-B6 materials have been used and tested in other applications, greater reliability is gained by using this approach.

Like DABL69, the new broad-group library uses the KAOS/LIB-V ${ }^{6}$ response functions instead of the response functions available in the AMPX master data library. At the time DABL69 was created, the $\mathrm{KAOS}^{7}$ data was known to be more accurate.

Section two of this report provides summary descriptions of the DABL69, VITAMIN-E ${ }^{8}$, and VITAMIN-B6 cross-section libraries, and a description of the new broad-group library. Section three discusses the prompt neutron and photon sources produced in the DABL69 group structure for the current Nagasaki dose calculations. 


\section{SUMMARY DESCRIPTION OF RELATED CROSS-SECTION LIBRARIES}

The DABL69, VITAMIN-E, and VITAMIN-B6 cross-section libraries are distributed by the Radiation Shielding Information Computational Center (RSICC) at ORNL. Each library is packaged together with a complete set of documentation as part of the RSICC Data Library Collection (DLC). The abstract from the documentation for each DLC package is replicated in the appendices to this report.

\section{DABL69}

The DABL69 energy group structure is a subset of the VITAMIN-E group structure and is tailored to air-over-ground environments. DABL69 contains 46 neutron and 23 photon energy groups. Angular distributions are approximated using a P5 Legendre expansion. As in DLC- $31^{9}$, the neutron groups are tailored to allow for the major peaks and valleys in the total neutron cross sections of nitrogen, oxygen, silicon, and iron. The photon groups are tailored to allow accurate calculation of pair production, annihilation photon transport, hydrogen capture, and backscatter photon transport. In the neutron energy range between 0.2 and $1.8 \mathrm{MeV}$, additional boundaries were added to the parent DLC-31/FEWG1 group structure to give better resolution in the region where the largest fraction of the total kerma frequently occurs. Eighty materials from the DLC-113C/VITAMIN-E library that were available in October 1987 are included as a first part of the DABL69 library. The cross-section for these materials were collapsed using the AMPX code system with the VITAMIN-E weighting functions. The second part of the DABL69 library contains eight additional materials including $\mathrm{N}-14, \mathrm{O}-16$, and steel using problem-dependent weighting functions generated by an ANISN analysis of a typical problem. Source spectra and response functions useful in radiation transport applications are included in the library. Elemental kerma factors are provided for nuclides ranging from $\mathrm{H}-1$ to Am-243. DABL69 is available as ASCII data for ANISN input. The kerma factor data are derived from DLC-160/KAOS-LIB-V. The DLC-130/DABL69 abstract is replicated in Appendix A. The DLC-160 abstract is replicated in Appendix B, and the DLC-160 description of the nuclear responses in KAOS-LIB-V is replicated in Appendix C.

\section{VITAMIN-E}

The energy structure of VITAMIN-E contains 174 neutron and 38 photon groups and includes the 171 neutron and 36 photon groups of VITAMIN-C ${ }^{10}$ as a subset. The group structure has fine detail in the energy region where cross-section minima occur for important shielding materials. The 174 neutron group data were processed with MINXI $5^{11}$; the 174 neutron group interaction data and the 38 group photon production data were processed with LAPHNGAS (AMPX III); and the 38 photon group data were processed with SMUG (AMPXIII) from DLC-99/HUGO ${ }^{12}$. The ENDF/B-V special purpose dosimetry, activation, and gas production files have also been processed into the VITAMINE group structure using XLACS2, NITAWL, and WORM. The DLC-113/VITAMIN-E abstract is replicated in Appendix D. 


\section{VITAMIN-B6}

VITAMIN-B6 is derived from ENDF/B-VI, Release 3, nuclear data, except for the Sn data that is obtained from LENDL ${ }^{13}$ and the Zirc2 data that is obtained from ENDF/B-IV. The responses and kerma factors were derived primarily from ENDF/B-VI. The ENDF data were processed with the PSR-355/NJOY $94^{14}$ code system and converted to the AMPX master library format with the SMILER module of PSR-315/AMPX77. The actual 199 neutron group boundaries in VITAMIN-B6 were selected from the 175 groups in VITAMIN-J ${ }^{15}$ (a European library based on the VITAMIN-C and VITAMIN-E structures) and the 27 groups used in the SCALE ${ }^{16}$ shielding library, with deference given to the VITAMIN-J boundaries at higher energies when the energy values are slightly different. The thermal energy range, which contains 36 neutron groups, is defined with $5.043 \mathrm{eV}$ as the uppermost boundary. The photon energy group structure is based on a combination of the 42 photon groups in VITAMIN-J and the 18 groups in the SCALE shielding library. The top energy group extends to $30 \mathrm{MeV}$, which allows proper representation of high energy photons from neutron capture at high energies. Although the cross section for capture at neutron energies between 20 and $30 \mathrm{MeV}$ is small, such a reaction in some materials could produce photons with energies between 20 and $30 \mathrm{MeV}$ (VITAMIN-E photon groups have a maximum top energy boundary of 20 $\mathrm{MeV})$. The DLC-184/VITAMIN-B6 abstract is replicated in Appendix E.

\section{NEW LIBRARIES}

The new broad-group cross-section library created to estimate doses received by surviving Nagasaki factory workers has the DABL69 energy group structure and is based on ENDF/B-VI data. Most of the materials in DABL69 created with standard weighting were included in the new broad-group library. Ar, Sn, I-127, Cs-133, Cs-137, Gd, and Pd were not available in VITAMIN-B6 and are not in the new broad-group library. Also, data for multiple isotopes rather than the natural element are included for $\mathrm{Cr}, \mathrm{Fe}, \mathrm{Nr}, \mathrm{Cu}$, and $\mathrm{Pb}$. The neutron and photon energy group boundaries for VITAMIN-B6, VITAMIN-E, and DABL69 are provided in Table 1 and Table 2. The list of materials in DABL69 is provided in Table 3, and the list of materials in VITAMIN-B6 is provided in Table 4. The materials in the new broad-group library are listed in Table 5. Table 6 lists the materials having response functions in KAOS/LIB-V.

The order of scattering used for both neutrons and photons is $\mathrm{P}_{7}$ for nuclides having $\mathrm{Z}$ less than or equal to 29 and $\mathrm{P}_{5}$ for the remainder of nuclides for both the new fine-group library in the VITAMIN-B6 group structure and the new broad-group library in the DABL69 group structure. The standard weigting spectrum in VITAMIN-B6 was used to create the new broad-group library, and consists of a Maxwellian thermal spectrum for neutron energies less than $0.125 \mathrm{eV}$, a "1/E" slowingdown spectrum for neutron energies between $0.125 \mathrm{eV}$ and $820.8 \mathrm{keV}$, and a fission spectrum for neutron energies between $820.8 \mathrm{keV}$ and $20 \mathrm{MeV}$. The VITAMIN-B6 standard weighting spectrum corresponds to IWT $=3$ in the NJOY ${ }^{14}$ module GAMINR (1/E plus roll offs).

The fine-group library has 199 neutron groups and 42 photon groups. The cross-section table length 
of 279 positions provides for 240 downscatters and 35 upscatters, with group 164 being the first thermal neutron group. Upscatter has not been included in the new broad-group library. 


\section{DESCRIPTION OF NEUTRON AND PHOTON SOURCE SPECTRA}

The Nagasaki source term is presented in open literature ${ }^{17}$, and is repeated in Tables 7 and 8 as the "literature" values. The literature presents the source term as yield per kiloton, with an energy value corresponding to each yield/kiloton value. It is assumed that the energy value is an upper bound on an energy interval, with the next lowest given energy as the lower bound of the energy interval. It is also assumed that the yield per kiloton value, per unit energy, is constant over the energy interval.

When radiation transport analyses are performed, a source term is prepared and input to the calculation in the same group structure as the cross sections. The space- and energy- dependent source is then integrated over all energy and volume and normalized, in 1D (ANISN) and 2D $(\text { DORT })^{18}$ analyses, to an input parameter. The 3D (TORT) ${ }^{19}$ code employs a multiplier, so the user must choose the multiplier with care for proper normalization. Consequently, the source term prepared for the DABL69 group structure should have the same integral over energy as the source provided in the literature.

The "calculated" source terms in Tables 7 and 8 are the source terms for the DABL69 energy group structure. They were calculated by the INTERP program written by, and obtained from, Dr. Richard A. Lillie at ORNL. This program was undocumented, but work has recently been performed to expand the capabilities of this program. The option to interpolate a flux was specified, along with energy values and the reference source term values, as input. The output of this program is listed in Tables 3 and 4 as the "calculated" values.

The neutron and photon source terms are depicted in Figures 1 and 2, respectively. To compare the "literature" and "calculated" values meaningfully, the source term values were divided by the width of the energy interval prior to being plotted. 


\section{REFERENCES}

1. P. F. Rose and C. L. Dunford, "Data Formats and Procedures for the Evaluated Nuclear Data File ENDF-6,” BNL-NCS-44945 (ENDF 102), (July 1990).

2. J. E. White et. al., "VITAMIN-B6: A Fine-Group Cross Section Library Based on ENDF/B-VI for Radiation Transport Applications," International Conference on Nuclear Data for Science and Technology, Gatlinburg, TN, p. 733, American Nuclear Society, LaGrange Park, IL, (1994).

3. N. M. Greene, “AMPX-77: A Modular Code System for Generating Coupled Multigroup Neutron-Gamma Cross Section Libraries from ENDF/B-IV and/or ENDF/B-V," ORNL/CSD/TM-283.

4. W. W. Engle, Jr. et. al., "A User's Manual for ANISN - A One-Dimensional Discrete Ordinates Transport Code with Anisotropic Scattering," Oak Ridge Gaseous Diffusion Plant Report, K-1693 (1967).

5. D. T. Ingersoll et. al., "DABL69: A Broad-Group Neutron/Photon Cross-Section Library for Defense Nuclear Applications," ORNL/TM-10568, Oak Ridge National Laboratory, (1989).

6. Y. Farawila et. al., "A Library of Nuclear Response Functions Generated by KAOS-V Code from ENDF/B-V and Other Data Files," ANL/FPP/TM-241, Argonne National Laboratory, (April 1989). [Also available from RSICC as DLC-160 (KAOS/LIB-V)]

7. Y. Farawila et. al., "KAOS-V Code: An Evaluation Tool for Neutron Kerma Factors and Other Nuclear Responses," ANL/FPP/TM-240, Argonne National Laboratory, (September 1989). [Also available from RSICC as PSR-306.]

8. C. R. Weisbin, et. al., "VITAMIN-E: An ENDF/B-V Multigroup Cross Section Library for LMFBR Core and Shield, LWR Shield, Dosimetry, and Fusion Blanket Technology," ORNL-5505, (February 1979).

9. D. E. Bartine et. al., "Production and Testing of the DNA Few-Group Coupled NeutronGamma Cross-Section Library," ORNL/TM-4840, Oak Ridge National Laboratory, (1977).

10. R. W. Roussin et. al., "VITAMIN-C: The CTR Processed Multigroup Cross Section Library for Neutronics Studies," ORNL/RSIC-37, Oak Ridge National Laboratory, (1980). [Also available from RSICC as DLC-41/VITAMIN-C]

11. J. E. White, et al, "MINX I.5: User's Manual for the ENDF/B-V IBM Version of the 
MINX Cross Section Processing Program,” unpublished.

12. R. W. Roussin et. al., "Description of the DLC-99/HUGO Package of Photon Interaction Data in ENDF/B-V Format," ORNL/RSIC-46 (ENDF-335), (December 1983).

13. R. J. Howerton, D. C. Cullen, M. H. MacGregor, S. T. Perkins, and E. F. Plechaty, "The LLL Evaluated-Nuclear-Data-Library (ENDL): Graphs of Cross Sections from the Library," UCRL-50400, Vol. 15 (October 1978). [Also available from RSICC as DLC034 (LENDL)]

14. R. E. MacFarlane et. al., "NJOY94.10: Code System for Producing Pointwise and Multigroup Neutron and Photon Cross Sections from ENDF/B Data," RSIC Computer Code Collection, PSR-355, (August 1995).

15. E. Sartori, "VITAMIN-J, A 175 Group Neutron Cross Section Library based on JEF-1 for Shielding Benchmark Calculations," JEF/DOC-100, (1985).

16. Oak Ridge National Laboratory, "SCALE: A Modular Code System for Performing Standardized Computer Analyses for Licensing Evaluation," NUREG/CR-200, Revision 4 (ORNL/NUREG/CSD-2/R4), Vols. I, II, and III, (April 1995).

17. William C. Roesch, Ed., "Reassessment of Atomic Bomb Radiation Dosimetry in Hiroshima and Nagasaki," The Radiation Effects Research Foundation, Japan (1987).

18. W. A. Rhoades and R. L. Childs, "DORT: A Two-Dimensional Discrete Ordinates Transport Code," in A User's Manual for MASH v2.0 - A Monte Carlo Adjoint Shielding Code System, J. O. Johnson, Ed., ORNL/TM-11788/R2, Oak Ridge National Laboratory, (May 1999).

19. W. A. Rhoades et. al., "The TORT Three-Dimensional Discrete Ordinates Neutron/Photon Transport Code (TORT Version 3)," ORNL/TM-13221, (October 1977). 
Table 1. Energy Boundaries for Neutron Groups

\begin{tabular}{|c|c|c|c|}
\hline & \multicolumn{3}{|c|}{ Group Number } \\
\hline Upper Energy $(\mathrm{eV})$ & $\begin{array}{c}\text { VITAMIN- } \\
\text { B6 }\end{array}$ & $\begin{array}{l}\text { VITAMIN- } \\
\text { E }\end{array}$ & DABL69 \\
\hline $1.9640 \mathrm{e}+7$ & 1 & 1 & 1 \\
\hline $1.7332 \mathrm{e}+7$ & 2 & 2 & \\
\hline $1.6905 \mathrm{e}+7$ & 3 & 3 & 2 \\
\hline $1.6487 \mathrm{e}+7$ & 4 & 4 & \\
\hline $1.5683 \mathrm{e}+7$ & 5 & 5 & \\
\hline $1.4918 \mathrm{e}+7$ & 6 & 6 & 3 \\
\hline $1.4550 \mathrm{e}+7$ & 7 & 7 & \\
\hline $1.4191 \mathrm{e}+7$ & 8 & 8 & 4 \\
\hline $1.3840 \mathrm{e}+7$ & 9 & 9 & 5 \\
\hline $1.3499 \mathrm{e}+7$ & 10 & 10 & \\
\hline $1.2840 \mathrm{e}+7$ & 11 & & \\
\hline $1.2523 \mathrm{e}+7$ & 12 & 11 & 6 \\
\hline $1.2214 \mathrm{e}+7$ & 13 & 12 & 7 \\
\hline $1.1618 \mathrm{e}+7$ & 14 & 13 & \\
\hline $1.1052 \mathrm{e}+7$ & 15 & 14 & 8 \\
\hline $1.0513 \mathrm{e}+7$ & 16 & 15 & \\
\hline $1.0000 \mathrm{e}+7$ & 17 & 16 & 9 \\
\hline $9.5123 \mathrm{e}+6$ & 18 & 17 & \\
\hline $9.0484 \mathrm{e}+6$ & 19 & 18 & 10 \\
\hline $8.6071 \mathrm{e}+6$ & 20 & 19 & \\
\hline $8.1873 \mathrm{e}+6$ & 21 & 20 & 11 \\
\hline $7.7880 \mathrm{e}+6$ & 22 & 21 & \\
\hline $7.4082 \mathrm{e}+6$ & 23 & 22 & 12 \\
\hline $7.0469 \mathrm{e}+6$ & 24 & 23 & \\
\hline $6.7032 \mathrm{e}+6$ & 25 & 24 & \\
\hline $6.5924 \mathrm{e}+6$ & 26 & 25 & \\
\hline $6.3763 e+6$ & 27 & 26 & 13 \\
\hline $6.0653 \mathrm{e}+6$ & 28 & 27 & \\
\hline $5.7695 \mathrm{e}+6$ & 29 & 28 & \\
\hline $5.4881 \mathrm{e}+6$ & 30 & 29 & \\
\hline $5.2295 \mathrm{e}+6$ & 31 & 30 & \\
\hline $4.9659 \mathrm{e}+6$ & 32 & 31 & 14 \\
\hline $4.7237 \mathrm{e}+6$ & 33 & 32 & 15 \\
\hline $4.4933 \mathrm{e}+6$ & 34 & 33 & \\
\hline $4.0657 \mathrm{e}+6$ & 35 & 34 & 16 \\
\hline $3.6788 \mathrm{e}+6$ & 36 & 35 & \\
\hline $3.3287 \mathrm{e}+6$ & 37 & 36 & \\
\hline $3.1664 \mathrm{e}+6$ & 38 & 37 & \\
\hline $3.0119 \mathrm{e}+6$ & 39 & 38 & 17 \\
\hline $2.8651 \mathrm{e}+6$ & 40 & 39 & \\
\hline $2.7253 \mathrm{e}+6$ & 41 & 40 & \\
\hline
\end{tabular}


Table 1. Energy Boundaries for Neutron Groups

\begin{tabular}{|c|c|c|c|}
\hline & \multicolumn{3}{|c|}{ Group Number } \\
\hline Upper Energy (eV) & $\begin{array}{c}\text { VITAMIN- } \\
\text { B6 }\end{array}$ & $\begin{array}{c}\text { VITAMIN- } \\
\text { E }\end{array}$ & DABL69 \\
\hline $2.5924 \mathrm{e}+6$ & 42 & 41 & \\
\hline $2.4660 \mathrm{e}+6$ & 43 & 42 & \\
\hline $2.3852 \mathrm{e}+6$ & 44 & 43 & 18 \\
\hline $2.3653 \mathrm{e}+6$ & 45 & 44 & \\
\hline $2.3457 \mathrm{e}+6$ & 46 & 45 & \\
\hline $2.3069 \mathrm{e}+6$ & 47 & 46 & 19 \\
\hline $2.2313 \mathrm{e}+6$ & 48 & 47 & \\
\hline $2.1225 \mathrm{e}+6$ & 49 & 48 & \\
\hline $2.0190 \mathrm{e}+6$ & 50 & 49 & \\
\hline $1.9205 \mathrm{e}+6$ & 51 & 50 & \\
\hline $1.8268 \mathrm{e}+6$ & 52 & 51 & 20 \\
\hline $1.7377 \mathrm{e}+6$ & 53 & 52 & \\
\hline $1.6530 \mathrm{e}+6$ & 54 & 53 & \\
\hline $1.5724 \mathrm{e}+6$ & 55 & 54 & \\
\hline $1.4957 \mathrm{e}+6$ & 56 & 55 & \\
\hline $1.4227 \mathrm{e}+6$ & 57 & 56 & 21 \\
\hline $1.3534 \mathrm{e}+6$ & 58 & 57 & \\
\hline $1.2874 \mathrm{e}+6$ & 59 & 58 & \\
\hline $1.2246 \mathrm{e}+6$ & 60 & 59 & \\
\hline $1.1648 \mathrm{e}+6$ & 61 & 60 & \\
\hline $1.1080 \mathrm{e}+6$ & 62 & 61 & 22 \\
\hline $1.0026 \mathrm{e}+6$ & 63 & 62 & \\
\hline $9.6164 \mathrm{e}+5$ & 64 & 63 & 23 \\
\hline $9.0718 \mathrm{e}+5$ & 65 & 64 & \\
\hline $8.6294 \mathrm{e}+5$ & 66 & 65 & \\
\hline $8.2085 \mathrm{e}+5$ & 67 & 66 & 24 \\
\hline $7.8082 \mathrm{e}+5$ & 68 & 67 & \\
\hline $7.4274 \mathrm{e}+5$ & 69 & 68 & 25 \\
\hline $7.0651 \mathrm{e}+5$ & 70 & 69 & \\
\hline $6.7206 \mathrm{e}+5$ & 71 & 70 & \\
\hline $6.3928 \mathrm{e}+5$ & 72 & 71 & 26 \\
\hline $6.0810 \mathrm{e}+5$ & 73 & 72 & \\
\hline $5.7844 \mathrm{e}+5$ & 74 & 73 & \\
\hline $5.5023 e+5$ & 75 & 74 & 27 \\
\hline $5.2340 \mathrm{e}+5$ & 76 & 75 & \\
\hline $4.9787 \mathrm{e}+5$ & 77 & 76 & \\
\hline $4.5049 \mathrm{e}+5$ & 78 & 77 & \\
\hline $4.0762 \mathrm{e}+5$ & 79 & 78 & \\
\hline $3.8774 \mathrm{e}+5$ & 80 & 79 & \\
\hline $3.6883 \mathrm{e}+5$ & 81 & 80 & 28 \\
\hline $3.3373 \mathrm{e}+5$ & 82 & 81 & \\
\hline $3.0197 \mathrm{e}+5$ & 83 & 82 & \\
\hline $2.9849 \mathrm{e}+5$ & 84 & 83 & \\
\hline
\end{tabular}


Table 1. Energy Boundaries for Neutron Groups

\begin{tabular}{|c|c|c|c|}
\hline & \multicolumn{3}{|c|}{ Group Number } \\
\hline Upper Energy (eV) & $\begin{array}{c}\text { VITAMIN- } \\
\text { B6 }\end{array}$ & $\begin{array}{c}\text { VITAMIN- } \\
\text { E }\end{array}$ & DABL69 \\
\hline $2.9721 \mathrm{e}+5$ & 85 & 84 & \\
\hline $2.9452 \mathrm{e}+5$ & 86 & 85 & \\
\hline $2.8725 \mathrm{e}+5$ & 87 & 86 & \\
\hline $2.7324 \mathrm{e}+5$ & 88 & 87 & \\
\hline $2.4724 \mathrm{e}+5$ & 89 & 88 & 29 \\
\hline $2.3518 \mathrm{e}+5$ & 90 & 89 & \\
\hline $2.2371 \mathrm{e}+5$ & 91 & 90 & \\
\hline $2.1280 \mathrm{e}+5$ & 92 & 91 & \\
\hline $2.0242 \mathrm{e}+5$ & 93 & 92 & \\
\hline $1.9255 \mathrm{e}+5$ & 94 & 93 & \\
\hline $1.8316 \mathrm{e}+5$ & 95 & 94 & \\
\hline $1.7422 \mathrm{e}+5$ & 96 & 95 & \\
\hline $1.6573 e+5$ & 97 & 96 & \\
\hline $1.5764 \mathrm{e}+5$ & 98 & 97 & 30 \\
\hline $1.4996 \mathrm{e}+5$ & 99 & 98 & \\
\hline $1.4264 \mathrm{e}+5$ & 100 & 99 & \\
\hline $1.3569 \mathrm{e}+5$ & 101 & 100 & \\
\hline $1.2907 \mathrm{e}+5$ & 102 & 101 & \\
\hline $1.2277 \mathrm{e}+5$ & 103 & 102 & \\
\hline $1.1679 \mathrm{e}+5$ & 104 & 103 & \\
\hline $1.1109 \mathrm{e}+5$ & 105 & 104 & 31 \\
\hline $9.8037 \mathrm{e}+4$ & 106 & 105 & \\
\hline $8.6517 \mathrm{e}+4$ & 107 & 106 & \\
\hline $8.2503 \mathrm{e}+4$ & 108 & 107 & \\
\hline $7.9499 \mathrm{e}+4$ & 109 & 108 & \\
\hline $7.1998 \mathrm{e}+4$ & 110 & 109 & \\
\hline $6.7379 \mathrm{e}+4$ & 111 & 110 & \\
\hline $5.6562 \mathrm{e}+4$ & 112 & 111 & \\
\hline $5.2475 \mathrm{e}+4$ & 113 & 112 & 32 \\
\hline $4.6309 \mathrm{e}+4$ & 114 & 113 & \\
\hline $4.0868 \mathrm{e}+4$ & 115 & 114 & \\
\hline $3.4307 \mathrm{e}+4$ & 116 & 115 & 33 \\
\hline $3.1828 \mathrm{e}+4$ & 117 & 116 & \\
\hline $2.8501 \mathrm{e}+4$ & 118 & 117 & \\
\hline $2.7000 \mathrm{e}+4$ & 119 & 118 & \\
\hline $2.6058 \mathrm{e}+4$ & 120 & 119 & \\
\hline $2.4788 \mathrm{e}+4$ & 121 & 120 & 34 \\
\hline $2.4176 \mathrm{e}+4$ & 122 & 121 & \\
\hline $2.3579 \mathrm{e}+4$ & 123 & 122 & \\
\hline $2.1875 \mathrm{e}+4$ & 124 & 123 & 35 \\
\hline $1.9305 \mathrm{e}+4$ & 125 & 124 & \\
\hline $1.5034 \mathrm{e}+4$ & 126 & 125 & \\
\hline $1.1709 \mathrm{e}+4$ & 127 & 126 & \\
\hline
\end{tabular}


Table 1. Energy Boundaries for Neutron Groups

\begin{tabular}{|c|c|c|c|}
\hline & \multicolumn{3}{|c|}{ Group Number } \\
\hline Upper Energy (eV) & $\begin{array}{l}\text { VITAMIN- } \\
\text { B6 }\end{array}$ & $\begin{array}{l}\text { VITAMIN- } \\
\text { E }\end{array}$ & DABL69 \\
\hline $1.0595 e+4$ & 128 & 127 & 36 \\
\hline $9.1188 \mathrm{e}+3$ & 129 & 128 & \\
\hline $7.1017 \mathrm{e}+3$ & 130 & 129 & \\
\hline $5.5308 \mathrm{e}+3$ & 131 & 130 & \\
\hline $4.3074 \mathrm{e}+3$ & 132 & 131 & \\
\hline $3.7074 \mathrm{e}+3$ & 133 & 132 & \\
\hline $3.3546 \mathrm{e}+3$ & 134 & 133 & 37 \\
\hline $3.0354 \mathrm{e}+3$ & 135 & 134 & \\
\hline $2.7465 e+3$ & 136 & 135 & \\
\hline $2.6126 e+3$ & 137 & 136 & \\
\hline $2.4852 \mathrm{e}+3$ & 138 & 137 & \\
\hline $2.2487 \mathrm{e}+3$ & 139 & 138 & \\
\hline $2.0347 \mathrm{e}+3$ & 140 & 139 & \\
\hline $1.5846 \mathrm{e}+3$ & 141 & 140 & \\
\hline $1.2341 \mathrm{e}+3$ & 142 & 141 & 38 \\
\hline $9.6112 \mathrm{e}+2$ & 143 & 142 & \\
\hline $7.4852 \mathrm{e}+2$ & 144 & 143 & \\
\hline $5.8295 \mathrm{e}+2$ & 145 & 144 & 39 \\
\hline $4.5400 \mathrm{e}+2$ & 146 & 145 & \\
\hline $3.5357 \mathrm{e}+2$ & 147 & 146 & \\
\hline $2.7536 e+2$ & 148 & 147 & 40 \\
\hline $2.1445 \mathrm{e}+2$ & 149 & 148 & \\
\hline $1.6702 \mathrm{e}+2$ & 150 & 149 & \\
\hline $1.3007 \mathrm{e}+2$ & 151 & 150 & \\
\hline $1.0130 \mathrm{e}+2$ & 152 & 151 & 41 \\
\hline $7.8893 \mathrm{e}+1$ & 153 & 152 & \\
\hline $6.1442 \mathrm{e}+1$ & 154 & 153 & \\
\hline $4.7851 \mathrm{e}+1$ & 155 & 154 & \\
\hline $3.7266 \mathrm{e}+1$ & 156 & 155 & \\
\hline $2.9023 \mathrm{e}+1$ & 157 & 156 & 42 \\
\hline $2.2603 \mathrm{e}+1$ & 158 & 157 & \\
\hline $1.7604 \mathrm{e}+1$ & 159 & 158 & \\
\hline $1.3710 \mathrm{e}+1$ & 160 & 159 & \\
\hline $1.0677 \mathrm{e}+1$ & 161 & 160 & 43 \\
\hline $8.3153 \mathrm{e}+0$ & 162 & 161 & \\
\hline $6.4760 \mathrm{e}+0$ & 163 & 162 & \\
\hline $5.0435 \mathrm{e}+0$ & 164 & 163 & \\
\hline $3.9279 \mathrm{e}+0$ & 165 & 164 & \\
\hline $3.0590 \mathrm{e}+0$ & 166 & 165 & 44 \\
\hline $2.3824 \mathrm{e}+0$ & 167 & 166 & \\
\hline $1.8554 \mathrm{e}+0$ & 168 & 167 & \\
\hline $1.4450 \mathrm{e}+0$ & 169 & 168 & \\
\hline $1.3000 \mathrm{e}+0$ & 170 & & \\
\hline
\end{tabular}


Table 1. Energy Boundaries for Neutron Groups

\begin{tabular}{|c|c|c|c|}
\hline & \multicolumn{3}{|c|}{ Group Number } \\
\hline Upper Energy (eV) & $\begin{array}{l}\text { VITAMIN- } \\
\text { B6 }\end{array}$ & $\begin{array}{l}\text { VITAMIN- } \\
\text { E }\end{array}$ & DABL69 \\
\hline $1.1253 \mathrm{e}+0$ & 171 & 169 & 45 \\
\hline $1.0800 \mathrm{e}+0$ & 172 & & \\
\hline $1.0400 \mathrm{e}+0$ & 173 & & \\
\hline $1.0000 \mathrm{e}+0$ & 174 & & \\
\hline $8.7643 \mathrm{e}-1$ & 175 & 170 & \\
\hline $8.0000 \mathrm{e}-1$ & 176 & & \\
\hline $6.8256 \mathrm{e}-1$ & 177 & 171 & \\
\hline $6.2506 \mathrm{e}-1$ & 178 & & \\
\hline $5.3158 \mathrm{e}-1$ & 179 & 172 & \\
\hline $5.0000 \mathrm{e}-1$ & 180 & & \\
\hline $4.1399 \mathrm{e}-1$ & 181 & 173 & 46 \\
\hline $3.6680 \mathrm{e}-1$ & 182 & & \\
\hline $3.2500 \mathrm{e}-1$ & 183 & & \\
\hline $2.7500 \mathrm{e}-1$ & 184 & & \\
\hline $2.2500 \mathrm{e}-1$ & 185 & & \\
\hline $1.8400 \mathrm{e}-1$ & 186 & & \\
\hline $1.5000 \mathrm{e}-1$ & 187 & & \\
\hline $1.2500 \mathrm{e}-1$ & 188 & & \\
\hline $1.0000 \mathrm{e}-1$ & 189 & 174 & \\
\hline $7.0000 \mathrm{e}-2$ & 190 & & \\
\hline $5.0000 \mathrm{e}-2$ & 191 & & \\
\hline $4.0000 \mathrm{e}-2$ & 192 & & \\
\hline $3.0000 \mathrm{e}-2$ & 193 & & \\
\hline $2.1000 \mathrm{e}-2$ & 194 & & \\
\hline $1.4500 \mathrm{e}-2$ & 195 & & \\
\hline $1.0000 \mathrm{e}-2$ & 196 & & \\
\hline $5.0000 \mathrm{e}-3$ & 197 & & \\
\hline $2.0000 \mathrm{e}-3$ & 198 & & \\
\hline $5.0000 \mathrm{e}-4$ & 199 & & \\
\hline $1.0000 \mathrm{e}-5$ & & & \\
\hline
\end{tabular}


Table 2. Energy Boundaries for Photon Groups

\begin{tabular}{|c|c|c|c|}
\hline & \multicolumn{3}{|c|}{ Group Number } \\
\hline Upper Energy $(\mathrm{eV})$ & VITAMIN-B6 & VITAMIN-E & DABL-69 \\
\hline $3.00+7$ & 1 & & \\
\hline $2.00+7$ & 2 & 1 & 1 \\
\hline $1.40+7$ & 3 & 2 & 2 \\
\hline $1.20+7$ & 4 & 3 & 3 \\
\hline $1.00+7$ & 5 & 4 & 4 \\
\hline $8.00+6$ & 6 & 5 & 5 \\
\hline $7.50+6$ & 7 & 6 & \\
\hline $7.00+6$ & 8 & 7 & 6 \\
\hline $6.50+6$ & 9 & 8 & \\
\hline $6.00+6$ & 10 & 9 & 7 \\
\hline $5.50+6$ & 11 & 10 & \\
\hline $5.00+6$ & 12 & 11 & 8 \\
\hline $4.50+6$ & 13 & 12 & \\
\hline $4.00+6$ & 14 & 13 & 9 \\
\hline $3.50+6$ & 15 & 14 & \\
\hline $3.00+6$ & 16 & 15 & 10 \\
\hline $2.50+6$ & 17 & 16 & 11 \\
\hline $2.00+6$ & 18 & 17 & 12 \\
\hline $1.66+6$ & 19 & 18 & \\
\hline $1.50+6$ & 20 & 19 & 13 \\
\hline $1.34+6$ & 21 & & \\
\hline $1.33+6$ & 22 & 20 & \\
\hline $1.00+6$ & 23 & 21 & 14 \\
\hline $8.00+5$ & 24 & 22 & \\
\hline $7.00+5$ & 25 & 23 & 15 \\
\hline $6.00+5$ & 26 & 24 & \\
\hline $5.12+5$ & 27 & 25 & \\
\hline $5.10+5$ & 28 & 26 & \\
\hline $4.50+5$ & 29 & 27 & 16 \\
\hline $4.00+5$ & 30 & 28 & \\
\hline $3.00+5$ & 31 & 29 & 17 \\
\hline $2.00+5$ & 32 & 30 & \\
\hline $1.50+5$ & 33 & 31 & 18 \\
\hline $1.00+5$ & 34 & 32 & 19 \\
\hline $7.50+4$ & 35 & 33 & \\
\hline $7.00+4$ & 36 & 34 & 20 \\
\hline $6.00+4$ & 37 & 35 & \\
\hline $4.50+4$ & 38 & 36 & 21 \\
\hline $4.00+4$ & 39 & & \\
\hline $3.00+4$ & 40 & 37 & 22 \\
\hline $2.00+4$ & 41 & 38 & 23 \\
\hline $1.00+4$ & 42 & & \\
\hline $1.00+3$ & & & \\
\hline
\end{tabular}


TABLE 3. Materials in the DABL69 Library with Standard Weighting

\begin{tabular}{|c|c|c|c|c|c|}
\hline Material & ID (AMPX) & ID (ANISN) & Material & ID (AMPX) & ID (ANISN) \\
\hline $\mathrm{H}-1$ & 930101 & $1-6$ & Cs-137 & 966901 & $241-246$ \\
\hline $\mathrm{H}-2$ & 930202 & $7-12$ & Ba-138 & 135301 & $247-252$ \\
\hline H-3 & 116901 & $13-18$ & $\mathrm{Gd}$ & 8853 & $253-258$ \\
\hline He-4 & 127000 & $19-24$ & Hf-174 & 137401 & $259-264$ \\
\hline Li-6 & 130301 & $25-30$ & Hf-176 & 137601 & $265-270$ \\
\hline $\mathrm{Li}-7$ & 139701 & $31-36$ & Hf-177 & 137701 & $271-276$ \\
\hline Be-9 & 104 & $37-42$ & Hf-178 & 137801 & $277-282$ \\
\hline B-10 & 130501 & $43-48$ & Hf- 179 & 138301 & $283-288$ \\
\hline B-11 & 8811 & $49-54$ & Hf- 180 & 138401 & 289-294 \\
\hline $\mathrm{C}$ & 130601 & $55-60$ & Та-181 & 128502 & $295-300$ \\
\hline $\mathrm{N}-14$ & 127501 & $61-66$ & W-182 & 182 & $301-306$ \\
\hline O-16 & 127601 & $67-72$ & W-183 & 183 & $307-312$ \\
\hline F-19 & 130902 & 73-78 & W-184 & 184 & $313-318$ \\
\hline $\mathrm{Na}-23$ & 131101 & 79-84 & W-186 & 186 & $319-324$ \\
\hline $\mathrm{Mg}$ & 131201 & $85-90$ & Re-185 & 108301 & $325-330$ \\
\hline $\mathrm{Al}-27$ & 131301 & $91-96$ & Re-187 & 108401 & $331-336$ \\
\hline $\mathrm{Si}$ & 131401 & $97-102$ & $\mathrm{Pt}$ & 8860 & $337-342$ \\
\hline P-31 & 131501 & $103-108$ & Au-197 & 8861 & $343-348$ \\
\hline S & 134701 & $109-114$ & $\mathrm{~Pb}$ & 138202 & $349-354$ \\
\hline $\mathrm{Cl}$ & 114901 & $115-120$ & Bi-209 & 137501 & $355-360$ \\
\hline $\mathrm{Ar}$ & 8824 & $121-126$ & Th-232 & 139001 & $361-366$ \\
\hline K & 115001 & $127-132$ & $\mathrm{~Pa}-233$ & 139101 & $367-372$ \\
\hline $\mathrm{Ca}$ & 132003 & $133-138$ & U-233 & 139301 & $373-378$ \\
\hline $\mathrm{Ti}$ & 132201 & $139-144$ & U-234 & 9394 & $379-384$ \\
\hline V & 132301 & $145-150$ & U-235 & 139501 & $385-390$ \\
\hline $\mathrm{Cr}$ & 132401 & $151-156$ & U-236 & 139601 & $391-396$ \\
\hline Mn-55 & 132502 & $157-162$ & U-238 & 139801 & $397-402$ \\
\hline $\mathrm{Fe}$ & 923604 & $163-168$ & Np-237 & 133701 & $403-408$ \\
\hline Co-59 & 132703 & $169-174$ & $\mathrm{Pu}-238$ & 133801 & $409-414$ \\
\hline $\mathrm{Ni}$ & 132802 & $175-180$ & $\mathrm{Pu}-239$ & 139901 & $415-420$ \\
\hline $\mathrm{Cu}$ & 132901 & $181-186$ & $\mathrm{Pu}-240$ & 138001 & $421-426$ \\
\hline $\mathrm{Ga}$ & 135801 & $187-192$ & $\mathrm{Pu}-241$ & 138101 & $427-432$ \\
\hline Y-89 & 920201 & 193-198 & $\mathrm{Pu}-242$ & 134201 & $433-438$ \\
\hline $\mathrm{Zr}$ & 8841 & 199-204 & Am-241 & 136101 & $439-444$ \\
\hline Nb-93 & 118901 & $205-210$ & Am-242 & 854201 & $445-450$ \\
\hline Mo & 132101 & $211-216$ & Am-242m & 136901 & $451-456$ \\
\hline $\mathrm{Cd}$ & 8847 & $217-222$ & $\mathrm{Am}-243$ & 136301 & $457-462$ \\
\hline Sn & 8850 & $223-228$ & $\mathrm{Cm}-242$ & 864201 & $463-468$ \\
\hline I-127 & 960601 & $229-234$ & $\mathrm{Cm}-243$ & 134301 & $469-474$ \\
\hline Cs- 133 & 135501 & $235-240$ & $\mathrm{Cm}-244$ & 134401 & $475-480$ \\
\hline
\end{tabular}


Table 4. Nuclides Available in VITAMIN-B6

\begin{tabular}{|c|c|c|c|c|c|}
\hline Entry & Identifier & Nuclide & Entry & Identifier & Nuclide \\
\hline 1 & 47107 & ag107 & 61 & 3007 & $\operatorname{li} 7$ \\
\hline 2 & 47109 & ag 109 & 62 & 12000 & $\mathrm{mg}$ \\
\hline 3 & 13027 & al27 & 63 & 25055 & mn55 \\
\hline 4 & 95241 & am241 & 64 & 42000 & mo \\
\hline 5 & 95242 & $\operatorname{am} 242$ & 65 & 7014 & n14 \\
\hline 6 & 95601 & $\mathrm{am} 242 \mathrm{~m}$ & 66 & 7015 & n15 \\
\hline 7 & 95243 & am243 & 67 & 11023 & na23 \\
\hline 8 & 79197 & au197 & 68 & 41093 & nb93 \\
\hline 9 & 5010 & b10 & 69 & 28058 & ni58 \\
\hline 10 & 5011 & b11 & 70 & 28060 & ni60 \\
\hline 11 & 56138 & ba138 & 71 & 28061 & ni61 \\
\hline 12 & 4009 & be 9 & 72 & 28062 & ni62 \\
\hline 13 & 4309 & be9(th) & 73 & 28064 & ni64 \\
\hline 14 & 83209 & bi209 & 74 & 93237 & np237 \\
\hline 15 & 6012 & $\mathrm{c}$ & 75 & 93238 & np238 \\
\hline 16 & 6312 & c (gph) & 76 & 93239 & np239 \\
\hline 17 & 20000 & $\mathrm{ca}$ & 77 & 8016 & o16 \\
\hline 18 & 48000 & cd(nat) & 78 & 8017 & o17 \\
\hline 19 & 17000 & cl(nat) & 79 & 15031 & p31 \\
\hline 20 & 96241 & $\mathrm{~cm} 241$ & 80 & 91231 & pa231 \\
\hline 21 & 96242 & $\mathrm{~cm} 242$ & 81 & 91233 & pa233 \\
\hline 22 & 96243 & $\mathrm{~cm} 243$ & 82 & 82206 & pb206 \\
\hline 23 & 96244 & $\mathrm{~cm} 244$ & 83 & 82207 & pb207 \\
\hline 24 & 96245 & $\mathrm{~cm} 245$ & 84 & 82208 & pb208 \\
\hline 25 & 96246 & $\mathrm{~cm} 246$ & 85 & 94236 & pu236 \\
\hline 26 & 96247 & $\mathrm{~cm} 247$ & 86 & 94237 & pu237 \\
\hline 27 & 96248 & $\mathrm{~cm} 248$ & 87 & 94238 & pu238 \\
\hline 28 & 27059 & $\operatorname{co5} 5$ & 88 & 94239 & pu239 \\
\hline 29 & 24050 & cr50 & 89 & 94240 & pu240 \\
\hline 30 & 24052 & $\operatorname{cr} 52$ & 90 & 94241 & pu241 \\
\hline 31 & 24053 & cr53 & 91 & 94242 & pu242 \\
\hline 32 & 24054 & cr54 & 92 & 94243 & pu243 \\
\hline 33 & 29063 & cu63 & 93 & 94244 & pu244 \\
\hline 34 & 29065 & cu65 & 94 & 75185 & re185 \\
\hline 36 & 63152 & eu152 & 96 & 16000 & $\mathrm{~s}$ \\
\hline 37 & 63153 & eul53 & 97 & 16032 & $\mathrm{~s} 32$ \\
\hline 38 & 63154 & eu154 & 98 & 14000 & si \\
\hline
\end{tabular}


Table 4. Continued

$\begin{array}{rrlrrl}\text { Entry } & \text { Identifier } & \text { Nuclide } & \text { Entry } & \text { Identifier } & \text { Nuclide } \\ 39 & 63155 & \text { eu155 } & 99 & 50000 & \text { sn(nat) } \\ 40 & 9019 & \text { f19 } & 100 & 73181 & \text { ta181 } \\ 41 & 26054 & \text { fe54 } & 101 & 73182 & \text { ta182 } \\ 42 & 26056 & \text { fe56 } & 102 & 90230 & \text { th230 } \\ 43 & 26057 & \text { fe57 } & 103 & 90232 & \text { th232 } \\ 44 & 26058 & \text { fe58 } & 104 & 22000 & \text { ti } \\ 45 & 31000 & \text { ga } & 105 & 92232 & \text { u232 } \\ 46 & 1001 & \text { hl(h2o) } & 106 & 92233 & \text { u233 } \\ 47 & 1901 & \text { h1(ch2) } & 107 & 92234 & \text { u234 } \\ 48 & 1002 & \text { h2(d2o) } & 108 & 92235 & \text { u235 } \\ 49 & 1003 & \text { h3 } & 109 & 92236 & \text { u236 } \\ 50 & 2003 & \text { he3 } & 110 & 92237 & \text { u237 } \\ 51 & 2004 & \text { he4 } & 111 & 92238 & \text { u238 } \\ 52 & 72174 & \text { hfl74 } & 112 & 23000 & \text { v } \\ 53 & 72176 & \text { hfl76 } & 113 & 74000 & \text { w(nat) } \\ 54 & 72177 & \text { hfl77 } & 114 & 74182 & \text { w182 } \\ 55 & 72178 & \text { hfl78 } & 115 & 74183 & \text { w183 } \\ 56 & 72179 & \text { hfl79 } & 116 & 74184 & \text { w184 } \\ 57 & 72180 & \text { hfl80 } & 117 & 74186 & \text { w186 } \\ 58 & 49000 & \text { in(nat) } & 118 & 39089 & \text { y89 } \\ 59 & 19000 & \mathrm{k} & 119 & 40000 & \text { zr } \\ 60 & 3006 & \text { li6 } & 120 & 40302 & \text { zirc2 }\end{array}$


Table 5. List of Materials in New Libraries

ANISN ID

1 po data for

2 pl data for

3 p2 data for

4 p3 data for

5 p4 data for

6 p5 data for

7 p6 data for

8 p7 data for

9 po data for

10 p1 data for

11 p2 data for

12 p3 data for

13 p4 data for

14 p5 data for

15 po data for

16 p1 data for

17 p2 data for

18 p3 data for

19 p4 data for

20 p5 data for

21 po data for

22 p1 data for

23 p2 data for

24 p3 data for

25 p4 data for

26 p5 data for

27 po data for

28 pl data for

29 p2 data for

30 p3 data for

31 p4 data for

32 p5 data for

33 po data for

34 pl data for

35 p2 data for

36 p3 data for

37 p4 data for

38 p5 data for

39 po data for

40 p1 data for

41 p2 data for

42 p3 data for

43 p4 data for

44 p5 data for

45 p6 data for

46 p7 data for

47 po data for

48 p1 data for

49 p2 data for

50 p3 data for

51 p4 data for

52 p5 data for

NUCLIDE DESCRIPTION

a) 27

a) 127

a 127

a 127

a 127

al2 7

a 127

a 127

am241

am241

am 241

am 241

am241

am241

am2 42

am 242

am 242

am 242

am 242

am 242

am $242 \mathrm{~m}$

am $242 \mathrm{~m}$

am $242 \mathrm{~m}$

am $242 \mathrm{~m}$

am $242 \mathrm{~m}$

am $242 \mathrm{~m}$

am 243

am 243

am 243

am 243

am 243

am 243

au197

au197

aul97

au197

au197

au197

b10

b10

b10

b10

b10

b10

b10

b10

b11

b11

b11

b11

b11

b11 v94.10 standard wgt e611325vb60003092095 v94.10 standard wgt e611325vb60003092095 v94.10 standard wgt e611325vb60003092095 v94.10 standard wgt e611325vb60003092095 v94.10 standard wgt e611325vb60003092095 v94.10 standard wgt e611325vb60003092095 v94.10 standard wgt e611325vb60003092095 v94.10 standard wgt e611325vb60003092095 v94.10. standard wgt e639543vb60003011896 v94.10 standard wgt e $639543 \mathrm{vb} 60003011896$ v94.10 standard wgt e639543vb60003011896 v94.10 standard wgt e639543vb60003011896 v94.10 standard wgt e639543vb60003011896 v94.10 standard wgt e639543vb60003011896 v91.94 standard wgt e629546vb60003030195 v91.94 standard wgt e629546vb60003030195 v91.94 standard wgt e629546vb60003030195 v91.94 standard wgt e629546vb60003030195 v91.94 standard wgt e629546vb60003030195 v91.94 standard wgt e $629546 \mathrm{vb} 60003030195$ v91.94 standard wgt e629547vb60003030195 v91.94 standard wgt e629547vb60003030195 v91.94 standard wgt e629547vb60003030195 v91.94 standard wgt e629547vb60003030195 v91.94 standard wgt e629547vb60003030195 v91.94 standard wgt e629547vb60003030195 v91.94 standard wgt e619549vb60003030195 v91.94 standard wgt e619549vb60003030195 v91.94 standard wgt e619549vb60003030195 v91.94 standard wgt e619549vb60003030195 v91.94 standard wgt e619549vb60003030195 v91.94 standard wgt e619549vb60003030195 v91.94 standard wgt e627925vb60003030195 v91.94 standard wgt e627925vb60003030195 v91.94 standard wgt e627925vb60003030195 v91.94 standard wgt e627925vb60003030195 v91.94 standard wgt e627925vb60003030195 v91.94 standard wgt e627925vb60003030195 v91.94 standard wgt e620525vb60003030195 v91.94 standard wgt e620525vb60003030195 v91.94 standard wgt e620525vb60003030195 v91.94 standard wgt e620525vb60003030195 v91.94 standard wgt e620525vb60003030195 v91.94 standard wgt e620525vb60003030195 v91.94 standard wgt e620525vb60003030195 v91.94 standard wgt e620525vb60003030195 v91.94 standard wgt e610528vb60003030195 v91.94 standard wgt e610528vb60003030195 v91.94 standard wgt e610528vb60003030195 v91.94 standard wgt e610528vb60003030195 v91.94 standard wgt e610528vb60003030195 v91.94 standard wgt e610528vb60003030195 ampx id

13027

ampx id 13027

ampx id 13027

ampx id 13027

ampx id 13027

ampx id 13027

ampx id 13027

ampx id 13027

ampx id 95241

ampx id 95241

ampx id 95241

ampx id 95241

ampx id 95241

ampx id 95241

ampx id 95242

ampx id 95242

ampx id 95242

ampx id 95242

ampx id 95242

ampx id 95242

ampx id 95601

ampx id 95601

ampx id 95601

ampx id 95601

ampx id 95601

ampx id 95601

ampx id 95243

ampx id 95243

ampx id 95243

ampx id 95243

ampx id 95243

ampx id 95243

ampx id 79197

ampx id 79197

ampx id 79197

ampx id 79197

ampx id 79197

ampx id 79197

ampx id 5010

ampx id 5010

ampx id 5010

ampx id 5010

ampx id 5010

ampx id 5010

ampx id 5010

ampx id 5010

ampx id 5011

ampx id 5011

ampx id 5011

ampx id 5011

ampx id 5011

ampx id 5011 
Table 5. Continued

53 p6 data for 54 p7 data for 55 po data for 56 p1 data for 57 p2 data for 58 p3 data for 59 p4 data for 60 p5 data for 61 po data for 62 p1 data for $63 \mathrm{p} 2$ data for 64 p3 data for 65 p4 data for 66 p5 data for 67 p6 data for 68 p7 data for 69 po data for 70 p1 data for 71 p2 data for 72 p3 data for 73 p4 data for 74 p5 data for 75 po data for 76 p1 data for 77 p2 data for 78 p3 data for 79 p4 data for 80 p5 data for 81 p6 data for 82 p7 data for 83 p0 data for 84 p1 data for 85 p2 data for 86 p3 data for 87 p4 data for 88 p5 data for 89 p6 data for 90 p7 data for 91 po data for 92 p1 data for 93 p2 data for 94 p3 data for 95 p4 data for 96 p5 data for 97 p6 data for 98 p7 data for 99 po data for 100 p1 data for 101 p2 data for 102 p3 data for 103 p4 data for 104 p5 data for 105 po data for $106 \mathrm{p} 1$ data for b11

b11

ba 138

ba 138

ba 138

ba138

ba 138

ba 138

be 9

be 9

be 9

be 9

beg

be 9

be 9

be 9

bi209

bi209

bi209

bi2 09

bi209

bi209

$c$

C

c

c

$c$

$\mathrm{c}$

c

C

C (gph)

$\begin{array}{ll}\text { c } & (g p h) \\ \text { c } & \text { (gph) }\end{array}$

C (gph)

c (gph)

$c$ c (gph) $\mathrm{ca}$ ca $\mathrm{ca}$ $\mathrm{ca}$ $\mathrm{ca}$ $\mathrm{ca}$ $\mathrm{ca}$ $\mathrm{ca}$ cd (nat) v91.94 standard wgt e604800vb60003030195 cd(nat) v91.94 standard wgt e604800vb60003030195 cd(nat) v91.94 standard wgt e604800vb60003030195 cd(nat) v91.94 standard wgt e604800vb60003030195 cd(nat) v91.94 standard wgt e604800vb60003030195 cd(nat) v91.94 standard wgt e604800vb60003030195 cl (nat) v91.94 standard wgt e601700vb60003030195 cl (nat) v91.94 standard wgt e601700vb60003030195 ampx id $\operatorname{ampx}$ id ampx id $\operatorname{ampx}$ id ampx id ampx id ampx id ampx id ampx id ampx id ampx id ampx id ampx id ampx id ampx id ampx id ampx id ampx id ampx id ampx id ampx id ampx id ampx id ampx id ampx id ampx id ampx id ampx id ampx id ampx id ampx id ampx id ampx id ampx id ampx id ampx id ampx id ampx id ampx id ampx id ampx id ampx id ampx id ampx id ampx id ampx id ampx id ampx id ampx id ampx id ampx id ampx id ampx id ampx id
5011 5011

56138

56138

56138

56138

56138

56138

4009

4009

4009

4009

4009

4009

4009

4009

83209

83209

83209

83209

83209

83209

6012

6012

6012

6012

6012

6012

6012

6012

6312

6312

6312

6312

6312

6312

6312

6312

20000

20000

20000

20000

20000

20000

20000

20000

48000

48000

48000

48000

48000

48000

17000

17000 


\section{Table 5. Continued}

107 p2 data for 108 p3 data for 109 p4 data for 110 p5 data for 111 p6 data for 112 p7 data for 113 po data for 114 pl data for 115 p2 data for 116 p3 data for 117 p4 data for 118 p5 data for 119 po data for 120 pl data for 121 p2 data for 122 p3 data for 123 p4 data for 124 p5 data for 125 po data for $126 \mathrm{p} 1$ data for 127 p2 data for 128 p3 data for 129 p4 data for 130 p5 data for 131 po data for 132 p1 data for 133 p2 data for 134 p3 data for 135 p4 data for 136 p5 data for 137 p6 data for 138 p7 data for 139 po data for 140 p1 data for 141 p2 data for 142 p3 data for 143 p4 data for 144 p5 data for 145 p6 data for 146 p7 data for 147 po data for $148 \mathrm{pl}$ data for 149 p2 data for 150 p3 data for 151 p4 data for 152 p5 data for 153 p6 data for 154 p7 data for 155 po data for 156 pl data for $157 \mathrm{p} 2$ data for 158 p3 data for 159 p4 data for 160 p5 data for cl(nat) v91.94 standard wgt e601700vb60003030195 cl (nat) v91.94 standard wgt e601700vb60003030195 cl (nat) v91.94 standard wgt e601700vb60003030195 cl (nat) v91.94 standard wgt e601700vb60003030195 cl (nat) v91.94 standard wgt e601700vb60003030195 cl (nat) v91.94 standard wgt e601700vb60003030195 cm242 v91.94 standard wgt e619631vb60003030195 cm242 v91.94 standard wgt e619631vb60003030195 cm242 v91.94 standard wgt e619631vb60003030195 cm242 v91.94 standard wgt e619631vb60003030195 cm242 v91.94 standard wgt e619631vb60003030195 cm242 v91.94 standard wgt e619631vb60003030195 cm243 v91.94 standard wgt e609634vb60003030195 cm243 v91.94 standard wgt e609634vb60003030195 cm243 v91.94 standard wgt e609634vb60003030195 cm243 v91.94 standard wgt e609634vb60003030195 cm243 v91.94 standard wgt e609634vb60003030195 cm243 v91.94 standard wgt e609634vb60003030195 cm244 v91.94 standard wgt e609637vb60003030195 cm244 v91.94 standard wgt e609637vb60003030195 cm244 v91.94 standard wgt e609637vb60003030195 cm244 v91.94 standard wgt e609637vb60003030195 cm244 v91.94 standard wgt e609637vb60003030195 cm244 v91.94 standard wgt e609637vb60003030195 cr50 v91.94 standard wgt e622425vb60003030195 cr50 v91.94 standard wgt e622425vb60003030195 cr50 v91.94 standard wgt e622425vb60003030195 cr50 v91.94 standard wgt e622425vb60003030195 cr50 v91.94 standard wgt e622425vb60003030195 cr50 v91.94 standard wgt e622425vb60003030195 cr50 v91.94 standard wgt e622425vb60003030195 cr50 v91.94 standard wgt e622425vb60003030195 cr52 v91.94 standard wgt e622431vb60003030195 cr52 v91.94 standard wgt e622431vb60003030195 cr52 v91.94 standard wgt e622431vb60003030195 cr52 v91.94 standard wgt e622431vb60003030195 cr52 v91.94 standard wgt e622431vb60003030195 cr52 v91.94 standard wgt e622431vb60003030195 cr52 v91.94 standard wgt e622431vb60003030195 cr52 v91.94 standard wgt e622431vb60003030195 cr53 v91.94 standard wgt e622434vb60003030195 cr53 v91.94 standard wgt e622434vb60003030195 cr53 v91.94 standard wgt e622434vb60003030195 cr53 v91.94 standard wgt e622434vb60003030195 cr53 v91.94 standard wgt e622434vb60003030195 cr53 v91.94 standard wgt e622434vb60003030195 cr53 v91.94 standard wgt e622434vb60003030195 cr53 v91.94 standard wgt e622434vb60003030195 cr54 v91.94 standard wgt e622437vb60003030195 cr54 v91.94 standard wgt e622437vb60003030195 cr54 v91.94 standard wgt e622437vb60003030195 cr54 v91.94 standard wgt e622437vb60003030195 cr54 v91.94 standard wgt e622437vb60003030195 $\operatorname{cr} 54$ ampx id ampx id ampx id ampx id ampx id ampx id ampx id ampx id ampx id ampx id ampx id ampx id ampx id ampx id ampx id ampx id ampx id ampx id ampx id ampx id ampx id ampx id ampx id ampx id ampx id $a \operatorname{mpx}$ id ampx id ampx id ampx id ampx id ampx id ampx id ampx id ampx id ampx id ampx id ampx id ampx id ampx id ampx id ampx id ampx id ampx id ampx id ampx id ampx id ampx id ampx id ampx id $\operatorname{ampx}$ id ampx id ampx id ampx id ampx id
17000 17000 17000 17000 17000 17000 96242 96242 96242 96242 96242 96242 96243 96243 96243 96243 96243 96243 96244 96244 96244 96244 96244 96244 24050 24050 24050 24050 24050 24050 24050 24050 24052 24052 24052 24052 24052 24052

24052

24052

24053 24053

24053

24053

24053

24053

24053

24053

24054

24054

24054 24054 24054 24054 
Table 5. Continued

161 p6 data for 162 p7 data for 163 po data for 164 p1 data for 165 p2 data for 166 p3 data for 167 p4 data for 168 p5 data for 169 p6 data for 170 p7 data for 171 po data for 172 pl data for 173 p2 data for 174 p3 data for 175 p4 data for 176 p5 data for 177 p6 data for 178 p7 data for 179 po data for $180 \mathrm{p} 1$ data for 181 p2 data for 182 p3 data for 183 p4 data for 184 p5 data for 185 p6 data for 186 p7 data for 187 po data for 188 pl data for 189 p2 data for 190 p3 data for 191 p4 data for 192 p5 data for 193 p6 data for 194 p7 data for 195 po data for 196 p1 data for 197 p2 data for 198 p3 data for 199 p4 data for 200 p5 data for 201 p6 data for 202 p7 data for 203 po data for 204 pl data for 205 p2 data for 206 p3 data for 207 p4 data for 208 p5 data for 209 p6 data for 210 p7 data for 211 po data for 212 pl data for 213 p2 data for 214 p3 data for cr54

cr54

cu63

cu63

cu63

cu63

cu63

cu63

cu63

cu63

cu65

cu65

cu65

cu65

cu65

cu65

cu65

cu65

f19

f19

f19

f 19

f19

f19

f 19

f19

fe 54

fe 54

fe 54

fe 54

fe 54

fes4

fe 54

fe 54

fe56

fe56

fe56

fe56

fe56

fe56

fe56

fe56

fe57

fe 57

fe 57

fe57

fe57

fe57

fe 57

fe 57

fe 58

fe 58

fe58

fe58 v91.94 standard wgt e622437vb60003030195 v91.94 standard wgt e622437vb60003030195 v91.94 standard wgt e632925vb60003030195 v91.94 standard wgt e632925vb60003030195 v91.94 standard wgt e632925vb60003030195 v91.94 standard wgt e632925vb60003030195 v91.94 standard wgt e632925vb60003030195 v91.94 standard wgt e632925vb60003030195 v91.94 standard wgt e632925vb60003030195 v91.94 standard wgt e632925vb60003030195 v91.94 standard wgt e632931vb60003030195 v91.94 standard wgt e632931vb60003030195 v91.94 standard wgt e632931vb60003030195 v91.94 standard wgt e632931vb60003030195 v91.94 standard wgt e632931vb60003030195 v91.94 standard wgt e632931vb60003030195 v91.94 standard wgt e632931vb60003030195 v91.94 standard wgt e632931vb60003030195 v91.94 standard wgt e610925vb60003030195 v91.94 standard wgt e610925vb60003030195 v91.94 standard wgt e610925vb60003030195 v91.94 standard wgt e610925vb60003030195 v91.94 standard wgt e610925vb60003030195 v91.94 standard wgt e610925vb60003030195 v91.94 standard wgt e610925vb60003030195 v91.94 standard wgt e610925vb60003030195 v91.94 standard wgt e622625vb60003030195 v91.94 standard wgt e622625vb60003030195 v91.94 standard wgt e622625vb60003030195 v91.94 standard wgt e622625vb60003030195 v91.94 standard wgt e622625vb60003030195 v91.94 standard wgt e622625vb60003030195 v91.94 standard wgt e622625vb60003030195 v91.94 standard wgt e622625vb60003030195 v91.94 standard wgt e622631vb60003030195 v91.94 standard wgt e622631vb60003030195 v91.94 standard wgt e622631vb60003030195 v91.94 standard wgt e622631vb60003030195 v91.94 standard wgt e622631vb60003030195 v91.94 standard wgt e622631vb60003030195 v91.94 standard wgt e622631vb60003030195 v91.94 standard wgt e622631vb60003030195 v91.94 standard wgt e622634vb60003030195 v91.94 standard wgt e622634vb60003030195 v91.94 standard wgt e622634vb60003030195 v91.94 standard wgt e622634vb60003030195 v91.94 standard wgt e622634vb60003030195 v91.94 standard wgt e622634 vb60003030195 v91.94 standard wgt e622634vb60003030195 v91.94 standard wgt e622634vb60003030195 v91.94 standard wgt e622637vb60003030195 v91.94 standard wgt e622637vb60003030195 v91.94 standard wgt e622637vb60003030195 v91.94 standard wgt e622637vb60003030195 ampx id ampx id ampx id ampx id ampx id ampx id ampx id ampx id ampx id ampx id ampx id ampx id ampx id ampx id $\operatorname{ampx}$ id ampx id $\operatorname{ampx}$ id ampx id ampx id ampx id ampx id ampx id ampx id ampx id ampx id ampx id ampx id ampx id ampx id ampx id ampx id ampx id ampx id $\operatorname{ampx}$ id ampx id ampx id ampx id ampx id ampx id ampx id ampx id ampx id ampx id ampx id ampx id ampx id ampx id ampx id ampx id ampx id ampx id ampx id ampx id ampx id
24054

24054 29063 29063 29063 29063 29063 29063 29063 29063 29065 29065 29065 29065 29065 29065 29065 29065 9019 9019 9019 9019 9019 9019 9019 9019

26054 26054 26054 26054 26054 26054 26054 26054 26056 26056 26056 26056 26056 26056 26056 26056 26057 26057 26057 26057 26057 26057 26057 26057 26058 26058 26058 26058 
Table 5. Continued

\begin{abstract}
215 p4 data for 216 p5 data for 217 p6 data for 218 p7 data for 219 po data for 220 p1 data for 221 p2 data for 222 p3 data for 223 p4 data for 224 p5 data for

fe58

fe 58

fe 58

fe58

ga

ga

ga

ga

ga

ga
\end{abstract} 225 po data for 226 p1 data for 227 p2 data for 228 p3 data for 229 p4 data for 230 p5 data for 231 p6 data for 232 p7 data for 233 po data for 234 p1 data for 235 p2 data for 236 p3 data for 237 p4 data for 238 p5 data for 239 p6 data for 240 p7 data for 241 po data for 242 p1 data for 243 p2 data for 244 p3 data for 245 p4 data for 246 p5 data for 247 p6 data for 248 p7 data for 249 po data for 250 p1 data for 251 p2 data for 252 p3 data for 253 p4 data for 254 p5 data for 255 p6 data for 256 p7 data for 257 po data for $258 \mathrm{pl}$ data for 259 p2 data for 260 p3 data for 261 p4 data for 262 p5 data for 263 p6 data for 264 p7 data for 265 po data for 266 p1 data for 267 p2 data for 268 p3 data for h1 (h2o) h1 (h2o) h1 (h2o) hl (h20) h1 (h2o) h1 (h2o) h1 (h2o) hI (h2o) h1 (ch2) h1 (ch2) h1 (ch2) h1 (ch2) h1 (ch2) h1 (ch2) h1 (ch2) h1 (ch2) h2 (d2o) h2 (d2o) h2 (d2o) h2 (d2o) h2 (d2o) h2 (d2o) h2 (d2o) h2 (d2o) h3

h3

h3

h3

h3

h3

h3

h3

he 4

he 4

he 4

he 4

he 4

he 4

he 4

he 4

hf 174

hf 174

hf 174

hf 174 v91.94 standard wgt e622637vb60003030195 v91.94 standard wgt e622637vb60003030195 v91.94 standard wgt e622637vb60003030195 v91.94 standard wgt e622637vb60003030195 v91.94 standard wgt e603100vb60003030195 v91.94 standard wgt e603100vb60003030195 v91.94 standard wgt e603100vb60003030195 v91.94 standard wgt e603100vb60003030195 v91.94 standard wgt e603100vb60003030195 v91.94 standard wgt e603100vb60003030195 v91.94 standard wgt e620125vb60003030195 v91.94 standard wgt e620125vb60003030195 v91.94 standard wgt e620125vb60003030195 v91.94 standard wgt e620125vb60003030195 v91.94 standard wgt e620125vb60003030195 v91.94 standard wgt e620125vb60003030195 v91.94 standard wgt e620125vb60003030195 v91.94 standard wgt e620125vb60003030195 v91.94 standard wgt e620137vb60003030195 v91.94 standard wgt e620137vb60003030195 v91.94 standard wgt e620137vb60003030195 v91.94 standard wgt e620137vb60003030195 v91.94 standard wgt e620137vb60003030195 v91.94 standard wgt e620137vb60003030195 v91.94 standard wgt e620137vb60003030195 v91.94 standard wgt e620137vb60003030195 v94.10 standard wgt e620128vb60003092095 v94.10 standard wgt e620128vb60003092095 v94.10 standard wgt e620128vb60003092095 v94.10 standard wgt e620128vb60003092095 v94.10 standard wgt e620128vb60003092095 v94.10 standard wgt e620128vb60003092095 v94.10 standard wgt e620128vb60003092095 v94.10 standard wgt e620128vb60003092095 v91.94 standard wgt e600131vb60003030195 v91.94 standard wgt e600131vb60003030195 v91.94 standard wgt e600131vb60003030195 v91.94 standard wgt e600131vb60003030195 v91.94 standard wgt e600131vb60003030195 v91.94 standard wgt e600131vb60003030195 v91.94 standard wgt e600131vb60003030195 v91.94 standard wgt e600131vb60003030195 v91.94 standard wgt e600228vb60003030195 v91.94 standard wgt e600228vb60003030195 v91.94 standard wgt e600228vb60003030195 v91.94 standard wgt e600228vb60003030195 v91.94 standard wgt e600228vb60003030195 v91.94 standard wgt e600228vb60003030195 v91.94 standard wgt e600228vb60003030195 v91.94 standard wgt e600228vb60003030195 v91.94 standard wgt e627225vb60003030195 v91.94 standard wgt e627225vb60003030195 v91.94 standard wgt e627225vb60003030195 v91.94 standard wgt e627225vb60003030195 ampx id ampx id ampx id ampx id ampx id $a \operatorname{mpx}$ id ampx id ampx id ampx id ampx id ampx id ampx id ampx id ampx id ampx id ampx id ampx id ampx id ampx id ampx id ampx id ampx id ampx id ampx id ampx id ampx id ampx id ampx id ampx id ampx id ampx id ampx id ampx id. ampx id $\operatorname{ampx}$ id ampx id ampx id ampx id ampx id ampx id ampx id ampx id $\operatorname{ampx}$ id ampx id ampx id ampx id ampx id ampx id ampx id ampx id ampx id ampx id ampx id ampx id
26058 26058 26058 26058 31000 31000 31000 31000 31000 31000

1001

1001

1001

1001

1001

1001

1001

1001

1901

1901

1901

1901

1901

1901

1901

1901

1002

1002

1002

1002

1002

1002

1002

1002

1003

1003

1003

1003

1003

1003

1003

1003

2004

2004

2004

2004

2004

2004

2004

2004

72174

72174

72174

72174 
Table 5. Continued

\begin{tabular}{|c|c|c|c|c|}
\hline 269 & $\mathrm{p} 4$ & data & for & $\mathrm{hf} 174$ \\
\hline 270 & $\mathrm{p} 5$ & data & for & $\operatorname{hf} 174$ \\
\hline 271 & po & data & for & $\operatorname{hf} 176$ \\
\hline 272 & $\mathrm{p} 1$ & data & for & $\operatorname{hf176}$ \\
\hline 273 & $\mathrm{p} 2$ & data & for & $h f 176$ \\
\hline 274 & p3 & data & for & $h f 176$ \\
\hline 275 & $\mathrm{p} 4$ & data & for & hf176 \\
\hline 276 & p5 & data & for & $h f 176$ \\
\hline 277 & po & data & for & $h f 177$ \\
\hline 278 & $\mathrm{p} 1$ & data & for & hf 177 \\
\hline 279 & $\mathrm{p} 2$ & data & for & hf 177 \\
\hline 280 & $\mathrm{p} 3$ & data & for & hf 177 \\
\hline 281 & $\mathrm{p} 4$ & data & for & $h f 177$ \\
\hline 282 & $\mathrm{p} 5$ & data & for & $\mathrm{hf} 177$ \\
\hline 283 & po & data & for & $h f 178$ \\
\hline 284 & $\mathrm{p} 1$ & data & for & $h f 178$ \\
\hline 285 & $\mathrm{p} 2$ & data & for & $\operatorname{hf} 178$ \\
\hline 286 & p3 & data & for & $\operatorname{hf} 178$ \\
\hline 287 & $\mathrm{p} 4$ & data & for & $\mathrm{hf} 178$ \\
\hline 288 & $\mathrm{p} 5$ & data & for & $h f 178$ \\
\hline 289 & po & data & for & $h f 179$ \\
\hline 290 & $\mathrm{p} 1$ & data & for & $\operatorname{hf} 179$ \\
\hline 291 & $\mathrm{p} 2$ & data & for & $\operatorname{hf} 179$ \\
\hline 292 & p3 & data & for & $h f 179$ \\
\hline 293 & $\mathrm{p} 4$ & data & for & $\mathrm{hf179}$ \\
\hline 294 & p 5 & data & for & $h f 179$ \\
\hline 295 & po & data & for & $\mathrm{hf} 180$ \\
\hline 296 & $\mathrm{p} 1$ & data & for & hf 180 \\
\hline 297 & $\mathrm{p} 2$ & data & for & $\mathrm{hf} 180$ \\
\hline 298 & $\mathrm{p} 3$ & data & for & $h f 180$ \\
\hline 299 & $\mathrm{p} 4$ & data & for & $\mathrm{hf} 180$ \\
\hline 300 & p5 & data & for & $h f 180$ \\
\hline 301 & po & data & for & $\mathrm{k}$ \\
\hline 302 & $\mathrm{p} 1$ & data & for & $\mathrm{k}$ \\
\hline 303 & $\mathrm{p} 2$ & data & for & $\mathrm{k}$ \\
\hline 304 & p3 & data & for & $\mathrm{k}$ \\
\hline 305 & $\mathrm{p} 4$ & data & for & $\mathrm{k}$ \\
\hline 306 & p5 & data & for & k \\
\hline 307 & p6 & data & for & k \\
\hline 308 & p7 & data & for & $\mathrm{k}$ \\
\hline 309 & po & data & for & $1 i 6$ \\
\hline 310 & $\mathrm{p} 1$ & data & for & 116 \\
\hline 311 & $\mathrm{p} 2$ & data & for & $1 i 6$ \\
\hline 312 & $\mathrm{p} 3$ & data & for & $1 i 6$ \\
\hline 313 & $\mathrm{p} 4$ & data & for & $1 i 6$ \\
\hline 314 & $\mathrm{p} 5$ & data & for & $1 i 6$ \\
\hline 315 & p6 & data & for & $1 i 6$ \\
\hline 316 & p7 & data & for & $1 i 6$ \\
\hline 317 & po & data & for & $1 i 7$ \\
\hline 318 & $\mathrm{p} 1$ & data & for & $1 i 7$ \\
\hline 319 & $\mathrm{p} 2$ & data & for & 117 \\
\hline 320 & p3 & data & for & $1 i 7$ \\
\hline 321 & $\mathrm{p} 4$ & data & for & $1 i 7$ \\
\hline 322 & $\mathrm{p} 5$ & data & for & $1 i 7$ \\
\hline
\end{tabular}

v91.94 standard wgt e627225vb60003030195 v91.94 standard wgt e627225vb60003030195 v91.94 standard wgt e627231vb60003030195 v91.94 standard wgt e627231vb60003030195 v91.94 standard wgt e627231vb60003030195 v91.94 standard wgt e627231vb60003030195 v91.94 standard wgt e627231vb60003030195 v91.94 standard wgt e627231vb60003030195 v91.94 standard wgt e627234vb60003030195 v91.94 standard wgt e627234vb60003030195 v91.94 standard wgt e627234vb60003030195 v91.94 standard wgt e627234 vb60003030195 v91.94 standard wgt e627234vb60003030195 v91.94 standard wgt e627234vb60003030195 v91.94 standard wgt e627237vb60003030195 v91.94 standard wgt e627237vb60003030195 v91.94 standard wgt e627237vb60003030195 v91.94 standard wgt e627237vb60003030195 v91.94 standard wgt e627237vb60003030195 v91.94 standard wgt e627237vb60003030195 v91.94 standard wgt e627240vb60003030195 v91.94 standard wgt e627240vb60003030195 v91.94 standard wgt e627240vb60003030195 v91.94 standard wgt e627240vb60003030195 v91.94 standard wgt e627240vb60003030195 v91.94 standard wgt e627240vb60003030195 v91.94 standard wgt e627243vb60003030195 v91.94 standard wgt e627243vb60003030195 v91.94 standard wgt e627243vb60003030195 v91.94 standard wgt e627243vb60003030195 v91.94 standard wgt e627243vb60003030195 v91.94 standard wgt e627243vb60003030195 v91.94 standard wgt e601900vb60003030195 v91.94 standard wgt e601900vb60003030195 v91.94 standard wgt e601900vb60003030195 v91.94 standard wgt e601900vb60003030195 v91.94 standard wgt e601900vb60003030195 v91.94 standard wgt e601900vb60003030195 v91.94 standard wgt e601900vb60003030195 v91.94 standard wgt e601900vb60003030195 v91.94 standard wgt e620325vb60003030195 v91.94 standard wgt e620325vb60003030195 v91.94 standard wgt e620325vb60003030195 v91.94 standard wgt e620325vb60003030195 v91.94 standard wgt e620325vb60003030195 v91.94 standard wgt e620325vb60003030195 v91.94 standard wgt e620325vb60003030195 v91.94 standard wgt e620325vb60003030195 v91.94 standard wgt e610328vb60003030195 v91.94 standard wgt e610328vb60003030195 v91.94 standard wgt e610328vb60003030195 v91.94 standard wgt e610328vb60003030195 v91.94 standard wgt e610328vb60003030195 v91.94 standard wgt e610328vb60003030195 ampx id ampx id ampx id ampx id ampx id ampx id ampx id ampx id ampx id ampx id ampx id ampx id ampx id ampx id ampx id ampx id ampx id ampx id ampx id ampx id ampx id ampx id ampx id ampx id ampx id ampx id ampx id ampx id ampx id ampx id ampx id ampx id ampx id ampx id ampx id ampx id ampx id ampx id ampx id ampx id ampx id ampx id ampx id $\operatorname{ampx}$ id ampx id ampx id ampx id $\operatorname{ampx} i d$ ampx id ampx id ampx id ampx id ampx id ampx id
72174 72174 72176 72176 72176 72176 72176 72176 72177 72177 72177 72177 72177 72177 72178 72178 72178 72178 72178 72178 72179 72179 72179 72179 72179 72179 72180 72180 72180 72180 72180 72180 19000 19000 19000 19000 19000 19000 19000 19000 3006 3006 3006 3006 3006 3006 3006 3006 3007 3007 3007 3007 3007 3007 
Table 5. Continued

\begin{tabular}{|c|c|c|c|c|c|c|c|c|}
\hline 23 & p6 & ta & or & $i 7$ & $\mathrm{v} 91.94$ & standard & wgt & e $610328 \mathrm{vb} 60003030195$ \\
\hline 24 & p7 & data & for & 117 & v91.94 & standard & wgt & e $610328 \mathrm{vb} 60003030195$ \\
\hline 25 & $\mathrm{p} 0$ & data & for & $\lg$ & v91.94 & standard & wgt & e $601200 \mathrm{vb} 60003030195$ \\
\hline 26 & $\mathrm{p} 1$ & data & for & ng & v91.94 & standard & wgt & e $601200 v b 60003030195$ \\
\hline & $\mathrm{p} 2$ & data & for & ng & $v 91.94$ & standard & wgt & e601200vb60003030195 \\
\hline 28 & p3 & data & for & $\mathrm{mg}$ & v91.94 & standard & wgt & e601200vb60003030195 \\
\hline 29 & $\mathrm{p} 4$ & data & for & $\mathrm{mg}$ & v91.94 & standard & & e601200vb60003030195 \\
\hline & p5 & data & for & $\mathrm{mg}$ & $v 91.94$ & standard & wgt & $\mathrm{e} 601200 \mathrm{vb} 6000303019 \mathrm{~s}$ \\
\hline 31 & p6 & data & for & $\mathrm{mg}$ & $v 91.94$ & standard & $g t$ & e601200vb60003030195 \\
\hline 32 & p7 & data & for & $\mathrm{mg}$ & v91.94 & standard & & e601200vb60003030195 \\
\hline 33 & po & data & for & $\operatorname{mn} 55$ & $\mathrm{v} 91.94$ & standard & $g t$ & e612525vb60003030195 \\
\hline 34 & $\mathrm{p} 1$ & data & for & $\operatorname{mn} 55$ & $v 91.94$ & stan & & e612525vb60003030195 \\
\hline 5 & $\mathrm{p} 2$ & data & for & $\operatorname{mn} 55$ & v91. 94 & stand & & e $612525 \mathrm{vb} 6000303019$ \\
\hline 6 & p3 & data & for & $\operatorname{mn} 55$ & v91.94 & stand & gt & e $612525 \mathrm{vb} 60003030195$ \\
\hline 37 & $\mathrm{p} 4$ & data & for & $\operatorname{mn} 55$ & v91.94 & star & & e612525vb60003030195 \\
\hline 38 & p5 & data & for & $\operatorname{mn} 55$ & $v 91.94$ & star & & e $612525 \mathrm{vb} 6000303019$ \\
\hline & p6 & data & for & $\operatorname{mn} 55$ & $v 91.94$ & sta & gt & e $612525 \mathrm{vb} 60003030195$ \\
\hline 10 & p7 & & for & $\operatorname{mn} 55$ & v91.94 & sta & & e $612525 \mathrm{vb} 6000303019$ \\
\hline & po & $: a$ & for & mo & $\mathrm{v} 91.94$ & sta & & e $604200 \mathrm{vb} 6000303019 \mathrm{~s}$ \\
\hline & $\mathrm{p} 1$ & data & for & mo & v91.94 & sta & gt & e $604200 \mathrm{vb} 60003030195$ \\
\hline$\$ 3$ & $\mathrm{p} 2$ & & For & mo & $v 91.94$ & sta & & e $604200 \mathrm{vb} 6000303019$ \\
\hline & p3 & data & for & mo & v91.94 & sta & & e $604200 \mathrm{vb} 6000303019$ \\
\hline & $\mathrm{p} 4$ & data & for & mo & $v 91.94$ & sta & $g t$ & e $604200 \mathrm{vb} 6000303019$ \\
\hline 6 & p5 & data & for & mo & v91.94 & sta & & e $604200 \mathrm{vb} 6000303019$ \\
\hline & po & & for & $\mathrm{n} 14$ & $\mathrm{v} 94.10$ & sta & & e630725vb60003092095 \\
\hline & $\mathrm{p} 1$ & & for & $\mathrm{n} 14$ & v94.10 & sta & t & e $630725 \mathrm{vb} 6000309209$ \\
\hline 19 & $\mathrm{p} 2$ & data & for & $\mathrm{n} 14$ & $\mathrm{v} 94.10$ & sta & 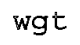 & e $630725 \mathrm{vb} 6 \mathrm{c}$ \\
\hline & p3 & & for & $\mathrm{n} 14$ & $\mathrm{v} 94.10$ & sta & & e630725vb60003092095 \\
\hline & $\mathrm{p} 4$ & & for & $\mathrm{n} 14$ & v94.10 & st & & e630725vb60003092095 \\
\hline 52 & $\mathrm{p} 5$ & data & for & $\mathrm{n} 14$ & $\mathrm{v} 94.10$ & sta &  & e $630725 \mathrm{vb} 60$ \\
\hline & p6 & & for & $\mathrm{n} 14$ & $\mathrm{v} 94.10$ & sta & gt & e630725vb60003092095 \\
\hline 4 & $\mathrm{p} 7$ & & for & $\mathrm{n} 14$ & $\mathrm{v} 94.10$ & sta & & e630725vb60003092095 \\
\hline 55 & $\mathrm{p} 0$ & data & for & na23 & v91.94 & sta & 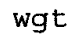 & e $621125 \mathrm{vb} 6 \mathrm{c}$ \\
\hline & $\mathrm{p} 1$ & & for & na23 & $v 91.94$ & sta & & e621125vb60003030195 \\
\hline 57 & $\mathrm{p} 2$ & & for & na23 & $\mathrm{v} 91.94$ & st & $g t$ & e621125vb6000303019 \\
\hline 58 & $\mathrm{p} 3$ & & for & $\operatorname{na} 23$ & $\mathrm{v} 91.94$ & & & e $621125 \mathrm{vb} 60003030195$ \\
\hline & $\mathrm{p} 4$ & & for & na23 & $\mathrm{v} 91.94$ & sta & & e $621125 \mathrm{vb} 6000303019$ \\
\hline 50 & $\mathrm{p} 5$ & data & for & $\operatorname{na} 23$ & $\mathrm{v} 91.94$ & sta & $3 \mathrm{c}$ & e621125vb60003030195 \\
\hline 51 & p6 & & for & na23 & $v 91.94$ & sta & & e $621125 \mathrm{vb} 6000303019$ \\
\hline 52 & $\mathrm{p} 7$ & & for & na23 & $\mathrm{v} 91.94$ & sta & t & e621125vb60003030195 \\
\hline 53 & po & data & for & $\mathrm{nb} 93$ & $\mathrm{v} 91.94$ & sta & gt & e $624125 \mathrm{vb} 6000303019$ \\
\hline 54 & $\mathrm{p} 1$ & & for & nb93 & v91.94 & sta & $g t$ & e $624125 \mathrm{vb} 60003030195$ \\
\hline 55 & $\mathrm{p} 2$ & & for & $\mathrm{nb} 93$ & $v 91.94$ & sta & t & e $624125 \mathrm{vb} 6000303019$ \\
\hline 56 & p3 & data & for & $\mathrm{nb} 93$ & v91.94 & sta & $g t$ & e $624125 \mathrm{vb} 6000303019$ \\
\hline 67 & $\mathrm{p} 4$ & & for & nb93 & v91.94 & sta & & e624125vb60003030195 \\
\hline 68 & $\mathrm{p} 5$ & data & for & nb93 & $v 91.94$ & sta & wgt & e $624125 \mathrm{vb} 60003030195$ \\
\hline 69 & po & data & for & ni58 & $\mathrm{v} 91.94$ & sta & & e $622825 \mathrm{vb} 60003030195$ \\
\hline 370 & $\mathrm{p} 1$ & & for & ni58 & $\mathrm{v} 91.94$ & sta & 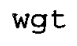 & e622825vb60003030195 \\
\hline 71 & $\mathrm{p} 2$ & data & for & ni58 & v91.94 & sta & $g t$ & e $622825 \mathrm{vb} 60003030195$ \\
\hline 372 & $\mathrm{p} 3$ & data & for & ni58 & v91. 94 & sta & & e $622825 \mathrm{vb} 60003030195$ \\
\hline 373 & $\mathrm{p} 4$ & da & for & ni58 & v91.94 & star & wg & e $622825 \mathrm{vb} 60003030195$ \\
\hline & p5 & & for & ni58 & v91.94 & star & $g \mathrm{c}$ & e $622825 \mathrm{vb} 6000303019$ \\
\hline & $\mathrm{p} 6$ & da & for & ni58 & $\mathrm{v} 91.94$ & stan & 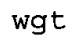 & e $622825 \mathrm{vb} 60003030195$ \\
\hline 76 & $\mathrm{p} 7$ & da & for & ni58 & v91.94 & standard & wgt & e $622825 \mathrm{vb} 6000303019$ \\
\hline
\end{tabular}

Ii 7

mg

mn 55

$\mathrm{mn} 55$

mn5 5

$\mathrm{mn} 55$

mn 55

mo

mo

n14

n14

na2 3

na 23

na2 3

na 23

nb93

nb93

ni58

ni58

ni58 v91.94 standard wgt e610328vb60003030195 .94 standard wgt e610328vb60003030195 v91.94 standard wgt e601200vb60003030195 v91.94 standard wgt e601200vb60003030195 030195 v91.94 standard wgt e601200vb60003030195 03030195 v91.94 standard wgt e612525vb60003030195 v91.94 standard wgt e612525vb60003030195 v91.94 standard wgt e612525vb60003030195 v91.94 standard wgt e604200vb60003030195 v91.94 standard wgt e604200vb60003030195 (1) 91.94 standard wgt e604200vb60003030195 v91.94 standard wgt e604200vb60003030195 (6) v94.10 standard wgt e630725vb60003092095 v4.10 standard Wgt $6630725 \mathrm{vb60003092095}$ v94.10 standaxd wgt e630725vb60003092095 v94.10 standard wgt e630725vb60003092095 v91. 94 standard v91.94 standard wgt e621125vb60003030195 v91.94 standard wgt e621125vb60003030195 v91.94 standard wgt e621125vb60003030195 v91.94 standard wgt e621125vb60003030195 60003030195 v91.94 standard wgt e624125vb60003030195 v91.94 standard wgt e624125vb60003030195 91. 94 standard wgt e624125vb60003030195 v91.94 standard wgt e624125vb60003030195 v91.94 standard wgt e624125vb60003030195 v91.94 standard wgt $622825 v b 60003030195$ v91.94 standard wgt e622825vb60003030195 v91.94 standard wgt e622825vb60003030195 v91.94 standard wgt e622825vb60003030195 v91.94 standard wgt e622825vb60003030195 v91. 94 standard wgt e612525vb60003030195 ampx id 3007 ampx id ampx id ampx id ampx id ampx id ampx id ampx id ampx id ampx id ampx id ampx id $\operatorname{ampx}$ id ampx id ampx id ampx id ampx id ampx id ampx id ampx id ampx id ampx id ampx id ampx id ampx id ampx id ampx id ampx id ampx id ampx id ampx id ampx id ampx id ampx id ampx id ampx id ampx id ampx id ampx id ampx id ampx id ampx id ampx id ampx id ampx id ampx id ampx id ampx id ampx id ampx id ampx id ampx id ampx id ampx id
3007

12000

12000

12000

12000

12000

12000

12000

12000

25055

25055

25055

25055

25055

25055

25055

25055

42000

42000

42000

42000

42000

42000

7014

7014

7014

7014

7014

7014

7014

7014

11023

11023

11023

11023

11023

11023

11023

11023

41093

41093

41093

41093

41093

41093

28058

28058

28058

28058

28058

28058

28058

28058 
Table 5. Continued

377 po data for 378 pl data for 379 p2 data for 380 p3 data for 381 p4 data for 382 p5 data for 383 p6 data for 384 p7 data for 385 po data for 386 p1 data for 387 p2 data for 388 p3 data for $389 \mathrm{p} 4$ data for 390 p5 data for 391 p6 data for 392 p7 data for 393 po data for $394 \mathrm{pl}$ data for 395 p2 data for 396 p3 data for 397 p4 data for 398 p5 data for 399 p6 data for 400 p7 data for 401 po data for 402 pl data for 403 p2 data for 404 p3 data for 405 p4 data for 406 p5 data for 407 p6 data for 408 p7 data for 409 po data for 410 p1 data for 411 p2 data for 412 p3 data for 413 p4 data for 414 p5 data for 415 po data for $416 \mathrm{pl}$ data for 417 p2 data for 418 p3 data for 419 p4 data for 420 p5 data for 421 p6 data for 422 p7 data for 423 po data for $424 \mathrm{pl}$ data for 425 p2 data for 426 p3 data for 427 p4 data for 428 p5 data for 429 p6 data for 430 p7 data for ni60

ni60

ni 60

ni60

ni 60

ni60

ni 60

ni60

ni61

ni 61

ni61

ni61

ni61

ni61

ni61

ni61

ni62

ni62

ni62

ni62

ni 62

ni62

ni62

ni62

ni64

ni64

ni64

ni64

ni 64

ni64

ni64

ni 64

np237

np237

np237

np237

np237

np237

016

016

016

016

016

016

016

016

p31

p31

p31

p31

p31

p31

p31

p31 v91.94 standard wgt e622831vb60003030195 v91.94 standard wgt e622831vb60003030195 v91.94 standard wgt e622831vb60003030195 v91.94 standard wgt e622831vb60003030195 v91.94 standard wgt e622831vb60003030195 v91.94 standard wgt e622831vb60003030195 v91.94 standard wgt e622831vb60003030195 v91.94 standard wgt e622831vb60003030195 v91.94 standard wgt e622834vb60003030195 v91.94 standard wgt e622834vb60003030195 v91.94 standard wgt e622834vb60003030195 v91.94 standard wgt e622834vb60003030195 v91.94 standard wgt e622834vb60003030195 v91.94 standard wgt e622834vb60003030195 v91.94 standard wgt e622834vb60003030195 v91.94 standard wgt e622834vb60003030195 v91.94 standard wgt e622837vb60003030195 v91.94 standard wgt e622837vb60003030195 v91.94 standard wgt e622837vb60003030195 v91.94 standard wgt e622837vb60003030195 v91.94 standard wgt e622837vb60003030195 v91.94 standard wgt e622837vb60003030195 v91.94 standard wgt e622837vb60003030195 v91.94 standard wgt e622837vb60003030195 v91.94 standard wgt e622843vb60003030195 v91.94 standard wgt e622843vb60003030195 v91.94 standard wgt e622843vb60003030195 v91.94 standard wgt e622843vb60003030195 v91.94 standard wgt e622843vb60003030195 v91.94 standard wgt e622843vb60003030195 v91.94 standard wgt e622843vb60003030195 v91.94 standard wgt e622843vb60003030195 v91.94 standard wgt e629346vb60003030195 v91.94 standard wgt e629346vb60003030195 v91.94 standard wgt e629346vb60003030195 v91.94 standard wgt e629346vb60003030195 v91.94 standard wgt e629346vb60003030195 v91.94 standard wgt e629346vb60003030195 v91.94 standard wgt e610825vb60003030195 v91.94 standard wgt e610825vb60003030195 v91.94 standard wgt e610825vb60003030195 v91.94 standard wgt e610825vb60003030195 v91.94 standard wgt e610825vb60003030195 v91.94 standard wgt e610825vb60003030195 v91.94 standard wgt e610825vb60003030195 v91.94 standard wgt e610825vb60003030195 v91.94 standard wgt e601525vb60003030195 v91.94 standard wgt e601525vb60003030195 v91.94 standard wgt e601525vb60003030195 v91.94 standard wgt e601525vb60003030195 v91.94 standard wgt e601525vb60003030195 v91.94 standard wgt e601525vb60003030195 v91.94 standard wgt e601525vb60003030195 v91.94 standard wgt e601525vb60003030195 ampx id ampx id ampx id ampx id $\operatorname{ampx}$ id ampx id ampx id ampx id ampx id ampx id ampx id ampx id ampx id ampx id ampx id ampx id ampx id $a \operatorname{mpx}$ id ampx id ampx id ampx id ampx id ampx id ampx id $\operatorname{ampx}$ id ampx id ampx id ampx id ampx id ampx id ampx id ampx id ampx id ampx id ampx id ampx id ampx id ampx id ampx id ampx id ampx id ampx id ampx id ampx id ampx id ampx id ampx id ampx id ampx id ampx id ampx id ampx id ampx id ampx id
28060 28060 28060 28060 28060 28060 28060 28060 28061 28061 28061 28061 28061 28061 28061 28061 28062 28062 28062 28062 28062 28062 28062 28062 28064 28064 28064 28064 28064 28064 28064 28064 93237 93237 93237 93237 93237 93237 8016 8016 8016 8016 8016 8016 8016 8016 15031 15031 15031 15031 15031 15031 15031 15031 
Table 5. Continued

431 po data for 432 pl data for 433 p2 data for 434 p3 data for $435 \mathrm{p} 4$ data for 436 p5 data for 437 po data for 438 p1 data for 439 p2 data for 440 p3 data for 441 p4 data for 442 p5 data for 443 po data for 444 p1 data for 445 p2 data for 446 p3 data for 447 p4 data for 448 p5 data for 449 po data for 450 p1 data for 451 p2 data for 452 p3 data for 453 p4 data for 454 p5 data for 455 po data for 456 pl data for 457 p2 data for 458 p3 data for 459 p4 data for 460 p5 data for 461 po data for 462 p1 data for 463 p2 data for 464 p3 data for 465 p4 data for 466 p5 data for 467 po data for 468 pl data for 469 p2 data for 470 p3 data for 471 p4 data for 472 p5 data for 473 po data for 474 p1 data for 475 p2 data for 476 p3 data for 477 p4 data for 478 p5 data for 479 po data for 480 p1 data for 481 p2 data for 482 p3 data for 483 p4 data for 484 p5 data for pa233

pa233

pa233

pa233

pa233

pa233

pb206

pb206

pb206

pb206

pb206

pb206

pb207

pb207

pb207

pb207

pb207

pb207

pb208

$\mathrm{pb} 208$

pb208

pb208

pb208

pb 208

pu238

pu238

pu23 8

pu238

pu238

pu238

pu239

pu239

pu239

pu239

pu239

pu239

pu240

pu240

pu240

pu240

pu240

pu240

pu24.1

pu241

pu241

pu241

pu241

pu241

pu242

pu 242

pu 242

pu 242

pu242

pu242 v91.94 standard wgt e609137vb60003030195 v91.94 standard wgt e609137vb60003030195 v91.94 standard wgt e609137vb60003030195 v91.94 standard wgt e609137vb60003030195 v91.94 standard wgt e609137vb60003030195 v91.94 standard wgt e609137vb60003030195 v91.94 standard wgt e618231vb60003030195 v91.94 standard wgt e618231vb60003030195 v91.94 standard wgt e618231vb60003030195 v91.94 standard wgt e618231vb60003030195 v91.94 standard wgt e618231vb60003030195 v91.94 standard wgt e618231vb60003030195 v91.94 standard wgt e628234vb60003030195 v91.94 standard wgt e628234vb60003030195 v91.94 standard wgt e628234vb60003030195 v91.94 standard wgt e628234vb60003030195 v91.94 standard wgt e628234vb60003030195 v91.94 standard wgt e628234vb60003030195 v91.94 standard wgt e618237vb60003030195 v91.94 standard wgt e618237vb60003030195 v91.94 standard wgt e618237vb60003030195 v91.94 standard wgt e618237vb60003030195 v91.94 standard wgt e618237vb60003030195 v91.94 standard wgt e618237vb60003030195 v91.94 standard wgt e619434vb60003030195 v91.94 standard wgt e619434vb60003030195 v91.94 standard wgt e619434vb60003030195 v91.94 standard wgt e619434vb60003030195 v91.94 standard wgt e619434vb60003030195 v91.94 standard wgt e619434vb60003030195 v91.94 standard wgt e629437vb60003030195 v91.94 standard wgt e629437vb60003030195 v91.94 standard wgt e629437vb60003030195 v91.94 standard wgt e629437vb60003030195 v91.94 standard wgt e629437vb60003030195 v91.94 standard wgt e629437vb60003030195 v91.94 standard wgt e639440vb60003030195 v91.94 standard wgt e639440vb60003030195 v91.94 standard wgt e639440vb60003030195 v91.94 standard wgt e639440vb60003030195 v91.94 standard wgt e639440vb60003030195 v91.94 standard wgt e639440vb60003030195 v94.10 standard wgt e639443vb60003092095 v94.10 standard wgt e639443vb60003092095 v94.10 standard wgt e639443vb60003092095 v94.10 standard wgt e639443vb60003092095 v94.10 standard wgt e639443vb60003092095 v94.10 standard wgt e639443vb60003092095 v91.94 standard wgt e619446vb60003030195 v91.94 standard wgt e619446vb60003030195 v91.94 standard wgt e619446vb60003030195 v91.94 standard wgt e619446vb60003030195 v91.94 standard wgt e619446vb60003030195 v91.94 standard wgt e619446vb60003030195 ampx id

ampx id

ampx id

ampx id

ampx id

ampx id

ampx id

ampx id

ampx id

ampx id

ampx id

ampx id

ampx id

ampx id

ampx id

ampx id

ampx id

ampx id

ampx id

ampx id

ampx id

ampx id

ampx id

ampx id

ampx id

ampx id

ampx id

ampx id

ampx id

ampx id

ampx id

ampx id

ampx id

ampx id

ampx id

ampx id

ampx id

ampx id

ampx id

ampx id

ampx id

ampx id

ampx id

$\operatorname{ampx}$ id

ampx id

ampx id

ampx id

ampx id

ampx id

ampx id

ampx id

ampx id

ampx id

ampx id
91233

91233

91233

91233

91233

91233

82206

82206

82206

82206

82206

82206

82207

82207

82207

82207

82207

82207

82208

82208

82208

82208

82208

82208

94238

94238

94238

94238

94238

94238

94239

94239

94239

94239

94239

94239

94240

94240

94240

94240

94240

94240

94241

94241

94241

94241

94241

94241

94242

94242

94242

94242

94242

94242 


\section{Table 5. Continued}

485 po data for $486 \mathrm{p} 1$ data for 487 p2 data for 488 p3 data for 489 p4 data for 490 p5 data for 491 po data for 492 pl data for 493 p2 data for 494 p3 data for 495 p4 data for 496 p5 data for 497 po data for 498 p1 data for 499 p2 data for 500 p3 data for 501 p4 data for 502 p5 data for 503 p6 data for 504 p7 data for 505 po data for 506 pl data for 507 p2 data for 508 p3 data for 509 p4 data for 510 p5 data for 511 p6 data for 512 p7 data for 513 po data for 514 p1 data for 515 p2 data for 516 p3 data for 517 p4 data for 518 p5 data for 519 po data for 520 p1 data for 521 p2 data for 522 p3 data for 523 p4 data for 524 p5 data for 525 po data for 526 p1 data for 527 p2 data for 528 p3 data for 529 p4 data for 530 p5 data for 531 po data for 532 pl data for 533 p2 data for 534 p3 data for 535 p4 data for 536 p5 data for 537 p6 data for 538 p7 data for re 185 re 185

re 185

re185

re 185

re185

re 187

re187

re 187

re 187

re 187

re 187

$\mathrm{s}$

$\mathrm{s}$

s

5

$\mathrm{s}$

$\mathbf{s}$

$\mathbf{s}$

$\mathrm{s}$

si

si

si

si

si

si

si

si

sn (nat) $\operatorname{sn}$ (nat) $\operatorname{sn}$ (nat) $\sin$ (nat) $\sin ($ nat) $\operatorname{sn}$ (nat)

ta181

ta181

ta181

ta181

ta181

ta181

th232

th232

th232

th232

th 232

th232

ti

$t i$

$t i$

ti

$t i$

ti

$t i$

$t i$ v91.94 standard wgt e617525vb60003030195 v91.94 standard wgt e617525vb60003030195 v91.94 standard wgt e617525vb60003030195 v91.94 standard wgt e617525vb60003030195 v91.94 standard wgt e617525vb60003030195 v91.94 standard wgt e617525vb60003030195 v91.94 standard wgt e617531vb60003030195 v91.94 standard wgt e617531vb60003030195 v91.94 standard wgt e617531vb60003030195 v91.94 standard wgt e617531vb60003030195 v91.94 standard wgt e617531vb60003030195 v91.94 standard wgt e617531vb60003030195 v91.94 standard wgt e601600vb60003030195 v91.94 standard wgt e601600vb60003030195 v91.94 standard wgt e601600vb60003030195 v91.94 standard wgt e601600vb60003030195 v91.94 standard wgt e601600vb60003030195 v91.94 standard wgt e601600vb60003030195 v91.94 standard wgt e601600vb60003030195 v91.94 standard wgt e601600vb60003030195 v91.94 standard wgt e601400vb60003030195 v91.94 standard wgt e601400vb60003030195 v91.94 standard wgt e601400vb60003030195 v91.94 standard wgt e601400vb60003030195 v91.94 standard wgt e601400vb60003030195 v91.94 standard wgt e601400vb60003030195 v91.94 standard wgt e601400vb60003030195 v91.94 standard wgt e601400vb60003030195 v91.94 standard wgt 1007850vb60003030195 v91.94 standard wgt $1007850 \mathrm{vb} 60003030195$ v91.94 standard wgt $1007850 \mathrm{vb} 60003030195$ v91.94 standard wgt 1007850vb60003030195 v91.94 standard wgt $1007850 \mathrm{vb} 60003030195$ v91.94 standard wgt $1007850 \mathrm{vb} 60003030195$ v91.94 standard wgt e607328vb60003030195 v91.94 standard wgt e607328vb60003030195 v91.94 standard wgt e607328vb60003030195 v91.94 standard wgt e607328vb60003030195 v91.94 standard wgt e607328vb60003030195 v91.94 standard wgt e607328vb60003030195 v91.94 standard wgt e619040vb60003030195 v91.94 standard wgt e619040vb60003030195 v91.94 standard wgt e619040vb60003030195 v91.94 standard wgt e619040vb60003030195 v91.94 standard wgt e619040vb60003030195 v91.94 standard wgt e619040vb60003030195 v91.94 standard wgt e602200vb60003030195 v91.94 standard wgt e602200vb60003030195 v91.94 standard wgt e602200vb60003030195 v91.94 standard wgt e602200vb60003030195 v91.94 standard wgt e602200vb60003030195 v91.94 standard wgt e602200vb60003030195 v91.94 standard wgt e602200vb60003030195 v91.94 standard wgt e602200vb60003030195 ampx id ampx id ampx id ampx id ampx id ampx id ampx id ampx id ampx id ampx id ampx id ampx id ampx id ampx id ampx id ampx id ampx id ampx id ampx id ampx id ampx id ampx id ampx id ampx id ampx id ampx id ampx id ampx id ampx id ampx id ampx id ampx id ampx id ampx id ampx id ampx id ampx id ampx id ampx id ampx id ampx id ampx id ampx id ampx id ampx id ampx id ampx id ampx id ampx id $a m p x$ id ampx id ampx id ampx id ampx id
75185 75185 75185 75185 75185 75185 75187 75187 75187 75187 75187 75187 16000 16000 16000 16000 16000 16000 16000 16000 14000 14000 14000 14000 14000 14000 14000 14000 50000 50000 50000 50000 50000 50000 73181 73181 73181 73181 73181 73181 90232 90232 90232 90232 90232 90232 22000 22000 22000 22000 22000 22000 22000 22000 


\section{Table 5. Continued}

539 po data for 540 p1 data for 541 p2 data for 542 p3 data for 543 p4 data for 544 p5 data for 545 po data for 546 pl data for 547 p2 data for 548 p3 data for 549 p4 data for 550 p5 data for 551 po data for 552 pl data for 553 p2 data for 554 p3 data for 555 p4 data for 556 p5 data for 557 po data for 558 p1 data for 559 p2 data for 560 p3 data for 561 p4 data for 562 p5 data for 563 po data for 564 pl data for 565 p2 data for 566 p3 data for 567 p4 data for 568 p5 data for 569 po data for 570 p1 data for 571 p2 data for 572 p3 data for 573 p4 data for 574 p5 data for 575 p6 data for 576 p7 data for 577 po data for 578 pl data for 579 p2 data for 580 p3 data for 581 p4 data for 582 p5 data for 583 po data for 584 p1 data for 585 p2 data for 586 p3 data for 587 p4 data for 588 p5 data for 589 po data for 590 p1 data for 591 p2 data for 592 p3 data for v91.94 standard wgt e619222vb60003030195 v91.94 standard wgt e619222vb60003030195 v91.94 standard wgt e619222vb60003030195 v91.94 standard wgt e619222vb60003030195 v91.94 standard wgt e619222vb60003030195 v91.94 standard wgt e619222vb60003030195 v91.94 standard wgt e619225vb60003030195 v91.94 standard wgt e619225vb60003030195 v91.94 standard wgt e619225vb60003030195 v91.94 standard wgt e619225vb60003030195 v91.94 standard wgt e619225vb60003030195 v91.94 standard wgt e619225vb60003030195 v94.10 standard wgt e649228vb60003092095 v94.10 standard wgt e649228vb60003092095 v94.10 standard wgt e649228vb60003092095 v94.10 standard wgt e649228vb60003092095 v94.10 standard wgt e649228vb60003092095 v94.10 standard wgt e649228vb60003092095 v91.94 standard wgt e619231vb60003030195 v91.94 standard wgt e619231vb60003030195 v91.94 standard wgt e619231vb60003030195 v91.94 standard wgt e619231vb60003030195 v91.94 standard wgt e619231vb60003030195 v91.94 standard wgt e619231vb60003030195 v91.94 standard wgt e639237vb60003030195 v91.94 standard wgt e639237vb60003030195 v91.94 standard wgt e639237vb60003030195 v91.94 standard wgt e639237vb60003030195 v91.94 standard wgt e639237vb60003030195 v91.94 standard wgt e639237vb60003030195 v91.94 standard wgt e612300vb60003030195 v91.94 standard wgt e612300vb60003030195 v91.94 standard wgt e612300vb60003030195 v91.94 standard wgt e612300vb60003030195 v91.94 standard wgt e612300vb60003030195 v91.94 standard wgt e612300vb60003030195 v91.94 standard wgt e612300vb60003030195 v91.94 standard wgt e612300vb60003030195 v91.94 standard wgt e607431vb60003030195 v91.94 standard wgt e607431vb60003030195 v91.94 standard wgt e607431vb60003030195 v91.94 standard wgt e607431vb60003030195 v91.94 standard wgt e607431vb60003030195 v91.94 standard wgt e607431vb60003030195 v91.94 standard wgt e607434vb60003030195 v91.94 standard wgt e607434vb60003030195 v91.94 standard wgt e607434vb60003030195 v91.94 standard wgt e607434vb60003030195 v91.94 standard wgt e607434vb60003030195 v91.94 standard wgt e607434vb60003030195 v91.94 standard wgt e607437vb60003030195 v91.94 standard wgt e607437vb60003030195 v91.94 standard wgt e607437vb60003030195 v91.94 standard wgt e607437vb60003030195 ampx id 92233 ampx id ampx id $\operatorname{ampx}$ id ampx id ampx id ampx id ampx id ampx id ampx id $\operatorname{ampx}$ id ampx id $\operatorname{ampx}$ id ampx id ampx id ampx id ampx id ampx id ampx id ampx id ampx id ampx id ampx id ampx id ampx id ampx id ampx id ampx id ampx id ampx id ampx id ampx id ampx id ampx id ampx id ampx id $\operatorname{ampx}$ id ampx id ampx id ampx id $a m p x$ id ampx id ampx id ampx id ampx id ampx id ampx id ampx id ampx id ampx id $a \operatorname{mpx}$ id ampx id ampx id ampx id
92233

92233

92233

92233

92233

92234

92234

92234

92234

92234

92234

92235

92235

92235

92235

92235

92235

92236

92236

92236

92236

92236

92236

92238

92238

92238

92238

92238

92238

23000

23000

23000

23000

23000

23000

23000

23000

74182

74182

74182

74182

74182

74182

74183

74183

74183

74183

74183

74183

74184

74184

74184

74184 


\section{Table 5. Continued}

\begin{tabular}{|c|c|c|c|c|}
\hline 993 & $\mathrm{p} 4$ & data & for & \\
\hline 594 & $\mathrm{p} 5$ & data & for & w1 \\
\hline 595 & po & data & for & \\
\hline 596 & $\mathrm{p} 1$ & data & for & \\
\hline 597 & $\mathrm{p} 2$ & data & for & \\
\hline 98 & p3 & data & for & $w \perp$ \\
\hline 99 & $\mathrm{p} 4$ & data & for & wI \\
\hline 600 & p5 & data & for & \\
\hline 01 & po & data & for & Y89 \\
\hline 602 & $\mathrm{p} 1$ & data & for & $y^{89}$ \\
\hline 603 & $\mathrm{p} 2$ & data & for & y89 \\
\hline 604 & p3 & data & for & $y^{89}$ \\
\hline 605 & $\mathrm{p} 4$ & data & for & $y^{89}$ \\
\hline 606 & p5 & data & for & y89 \\
\hline 607 & po & data & for & zr \\
\hline 608 & $\mathrm{p} 1$ & data & for & zr \\
\hline 609 & $\mathrm{p} 2$ & data & for & $\mathrm{zr}$ \\
\hline 61 & p3 & data & for & $\mathrm{zr}$ \\
\hline 61 & $\mathrm{p} 4$ & data & for & zr \\
\hline 61 & p5 & data & for & zr \\
\hline
\end{tabular}
v91.94 standard wgt e607437vb60003030195
v91.94 standard wgt e607437vb60003030195
v91.94 standard wgt e607443vb60003030195
ampx id
74184
v91.94 standard wgt e607443vb60003030195
ampx id
74184
ampx id 74186
v91.94 standard wgt e607443vb60003030195
v91.94 standard wgt e607443vb60003030195
v91.94 standard wgt e607443vb60003030195
v91.94 standard wgt e607443vb60003030195
v91.94 standard wgt e613925vb60003030195
v91.94 standard wgt e613925vb60003030195
v91.94 standard wgt e613925vb60003030195
v91.94 standard wgt e613925vb60003030195
v91.94 standard wgt e613925vb60003030195
v91.94 standard wgt e613925vb60003030195
v91.94 standard wgt e624000vb60003030195
v91.94 standard wgt e624000vb60003030195
v91.94 standard wgt e624000vb60003030195
v91.94 standard wgt e624000vb60003030195
v91.94 standard wgt e624000vb60003030195
v91.94 standard wgt e624000vb60003030195
ampx id 74186
ampx id 74186
ampx id 74186
ampx id 74186
ampx id 74186
ampx id 39089
ampx id 39089
ampx id 39089
ampx id 39089
ampx id 39089
ampx id 39089
ampx id 40000
ampx id 40000
ampx id 40000
ampx id 40000
ampx id 40000
ampx id 40000 
Table 6. List of Materials in KAOS/LIB-V

\begin{tabular}{|c|c|c|c|}
\hline No. & $\begin{array}{l}\text { Material } \\
\text { Name }\end{array}$ & $\begin{array}{l}\text { ENDF/B-V } \\
\text { MAT number }\end{array}$ & $\begin{array}{l}\text { KAOS/LIB-V } \\
\text { Symbol }\end{array}$ \\
\hline 1 & Hydrogen & 1301 & h-1 \\
\hline 2 & Deuterium & 1302 & h-2 \\
\hline 3 & Helium & 1270 & he-4 \\
\hline 4 & Lithium-6 & 1303 & li-6 \\
\hline 5 & Lithium-7 & 1397 & li-7 \\
\hline 6 & Beryllium & 1304 & be- 9 \\
\hline 7 & Boron-10 & 1305 & $b-10$ \\
\hline 8 & Boron-11 & 1160 & b-11 \\
\hline 9 & Carbon & 1306 & $\mathrm{c}-12$ \\
\hline 10 & Nitrogen & 1275 & $n-14$ \\
\hline 11 & Oxygen & 1276 & $0-16$ \\
\hline 12 & Fluorine & 1309 & $f-19$ \\
\hline 13 & Sodium & 1311 & na-23 \\
\hline 14 & Magnesium & 1312 & mg-nat \\
\hline 15 & Aluminum & 1313 & al-27 \\
\hline 16 & Silicon & 1314 & si-nat \\
\hline 17 & Phosphorus & 1315 & p-31 \\
\hline 18 & Sulfur & 1347 & s-nat \\
\hline 19 & Chlorine & 1149 & cl-nat \\
\hline 20 & Potassium & 1150 & k-nat \\
\hline 21 & Calcium & 1320 & ca-nat \\
\hline 22 & Titanium & 1322 & ti-nat \\
\hline 23 & Vanadium & 1323 & v-nat \\
\hline 24 & Chromium & 1324 & cr-nat \\
\hline 25 & Manganese & 1325 & $m n-55$ \\
\hline 26 & Iron & 1326 & fe-nat \\
\hline 27 & Cobalt & 1327 & co-59 \\
\hline 28 & Nickel & 1328 & ni-nat \\
\hline 29 & Copper & 1329 & cu-nat \\
\hline 30 & Zirconium-90 & 1385 & zr-90 \\
\hline 31 & Zirconium-91 & 1386 & zr-91 \\
\hline 32 & Zirconium-92 & 1387 & zr-92 \\
\hline 33 & Zirconium-94 & 1388 & zr-94 \\
\hline 34 & Zirconium-96 & 1389 & zr-96 \\
\hline
\end{tabular}


Table 6. List of Materials in KAOS/LIB-V (cont.)

\begin{tabular}{|c|c|c|c|}
\hline No. & $\begin{array}{l}\text { Material } \\
\text { Name }\end{array}$ & $\begin{array}{l}\text { ENDF/B-V } \\
\text { MAT number }\end{array}$ & $\begin{array}{l}\text { KAOS/LIB-V } \\
\text { Symbol }\end{array}$ \\
\hline 35 & Niobium & 1189 & nb-93 \\
\hline 36 & Molybdenum & 1321 & mo-nat \\
\hline 37 & Hafnium-174 & 1374 & hf-174 \\
\hline 38 & Hafnium-176 & 1376 & hf-176 \\
\hline 39 & Hafnium-177 & 1377 & hf-177 \\
\hline 40 & Hafnium-178 & 1378 & hf-178 \\
\hline 41 & Hafnium-179 & 1383 & hf-179 \\
\hline 42 & Hafnium-180 & 1384 & hf-180 \\
\hline 43 & Tantalum & 1285 & ta-181 \\
\hline 44 & Tungsten-182 & 1475 & w-182 \\
\hline 45 & Tungsten-183 & 1476 & w-183 \\
\hline 46 & Tungsten-184 & 1477 & w-184 \\
\hline 47 & Tungsten-186 & 1478 & w-186 \\
\hline 48 & Lead & 1382 & pb-nat \\
\hline 49 & Bismuth & 1375 & bi-209 \\
\hline 50 & Thorium-232 & 1390 & th-232 \\
\hline 51 & Protactinium-233 & 1391 & pa-233 \\
\hline 52 & Uranium-233 & 1393 & $\mathrm{u}-233$ \\
\hline 53 & Uranium-234 & 1394 & $\mathrm{u}-234$ \\
\hline 54 & Uranium-235 & 1395 & $\mathrm{u}-235$ \\
\hline 55 & Uranium-236 & 1396 & $\mathrm{u}-236$ \\
\hline 56 & Uranium-238 & 1398 & $\mathrm{u}-238$ \\
\hline 57 & Neptunium-237 & 1337 & np-237 \\
\hline 58 & Plutonium-238 & 1338 & pu-238 \\
\hline 59 & Plutonium-239 & 1399 & pu-239 \\
\hline 60 & Plutonium-240 & 1380 & $\mathrm{pu}-240$ \\
\hline 61 & Plutonium-241 & 1381 & pu-241 \\
\hline 62 & Plutonium-242 & 1342 & pu-242 \\
\hline 63 & Americium-241 & 1361 & am-241 \\
\hline 64 & Americium-243 & 1363 & am-243 \\
\hline
\end{tabular}


Table 7. Neutron Source Interpolation

Calculated

\begin{tabular}{|c|c|c|}
\hline Group & Energy(eV) & Yield per kiloton \\
\hline 1 & $1.96 e+07$ & \\
\hline 2 & $1.69 \mathrm{e}+07$ & $6.97 e-08$ \\
\hline 3 & $1.49 \mathrm{e}+07$ & $1.16 \mathrm{e}-07$ \\
\hline 4 & $1.42 \mathrm{e}+07$ & $6.67 e-08$ \\
\hline 5 & $1.38 \mathrm{e}+07$ & $7.54 \mathrm{e}-07$ \\
\hline 6 & $1.25 \mathrm{e}+07$ & $3.16 e-07$ \\
\hline 7 & $1.22 \mathrm{e}+07$ & $1.67 e-06$ \\
\hline 8 & $1.11 e+07$ & $2.25 \mathrm{e}-06$ \\
\hline 9 & $1.00 e+07$ & $5.75 e-06$ \\
\hline 10 & $9.05 e+06$ & $8.66 \mathrm{e}-06$ \\
\hline 11 & $8.19 \mathrm{e}+06$ & $1.40 \mathrm{e}-05$ \\
\hline 12 & $7.41 \mathrm{e}+06$ & $3.54 \mathrm{e}-05$ \\
\hline 13 & $6.38 e+06$ & $9.37 e-05$ \\
\hline 14 & $4.97 e+06$ & $1.79 \mathrm{e}-05$ \\
\hline 15 & $4.72 e+06$ & $7.90 e-05$ \\
\hline 16 & $4.07 e+06$ & $1.62 \mathrm{e}-04$ \\
\hline 17 & $3.01 e+06$ & $2.55 e-04$ \\
\hline 18 & $2.39 \mathrm{e}+06$ & $3.74 \mathrm{e}-05$ \\
\hline 19 & $2.31 e+06$ & $2.16 e-04$ \\
\hline 20 & $1.83 e+06$ & $1.91 e-04$ \\
\hline 21 & $1.42 \mathrm{e}+06$ & $1.58 \mathrm{e}-04$ \\
\hline 22 & $1.11 \mathrm{e}+06$ & $7.27 e-05$ \\
\hline 23 & $9.62 e+05$ & $6.94 \mathrm{e}-05$ \\
\hline 24 & $8.21 e+05$ & $5.25 e-05$ \\
\hline 25 & $7.43 e+05$ & $6.98 e-05$ \\
\hline 26 & $6.39 \mathrm{e}+05$ & $8.57 e-05$ \\
\hline 27 & $5.50 e+05$ & $1.30 \mathrm{e}-04$ \\
\hline 28 & $3.69 e+05$ & $9.06 e-05$ \\
\hline 29 & $2.47 e+05$ & $9.39 e-05$ \\
\hline 30 & $1.58 \mathrm{e}+05$ & $6.69 \mathrm{e}-05$ \\
\hline 31 & $1.11 e+05$ & $9.46 e-05$ \\
\hline 32 & $5.25 e+04$ & $3.86 e-05$ \\
\hline 33 & $3.43 e+04$ & $2.03 e-05$ \\
\hline 34 & $2.48 e+04$ & $1.28 e-05$ \\
\hline 35 & $2.19 e+04$ & $4.97 e-05$ \\
\hline 36 & $1.06 e+04$ & $8.86 e-05$ \\
\hline 37 & $3.35 e+03$ & $6.94 e-04$ \\
\hline 38 & $1.23 e+03$ & $3.64 e-02$ \\
\hline 39 & $5.83 e+02$ & $8.06 e-02$ \\
\hline 40 & $2.75 e+02$ & $8.77 e-02$ \\
\hline 41 & $1.01 e+02$ & $4.96 \mathrm{e}-02$ \\
\hline 42 & $2.90 \mathrm{e}+01$ & $1.19 e-02$ \\
\hline 43 & $1.07 e+01$ & $3.63 e-03$ \\
\hline 44 & $3.06 e+00$ & $4.42 \mathrm{e}-04$ \\
\hline 45 & $1.12 \mathrm{e}+00$ & $9.57 e-05$ \\
\hline 46 & $4.14 \mathrm{e}-01$ & $2.53 e-05$ \\
\hline & $1.00 \mathrm{e}-05$ & \\
\hline & sum $=$ & $2.73 e-01$ \\
\hline
\end{tabular}

Literature

$\begin{array}{cr}\text { Energy }(\mathrm{eV}) & \text { Yield per kiloton } \\ & \\ 1.60 \mathrm{e}+07 & 9.66 \mathrm{e}-08 \\ 1.45 \mathrm{e}+07 & 1.44 \mathrm{e}-07 \\ 1.40 \mathrm{e}+07 & 3.65 \mathrm{e}-08 \\ 1.35 \mathrm{e}+07 & 4.97 \mathrm{e}-07 \\ 1.28 \mathrm{e}+07 & 7.67 \mathrm{e}-07 \\ 1.20 \mathrm{e}+07 & 1.53 \mathrm{e}-06 \\ 1.10 \mathrm{e}+07 & 2.17 \mathrm{e}-06 \\ 1.00 \mathrm{e}+07 & 7.07 \mathrm{e}-06 \\ 8.83 \mathrm{e}+06 & 1.19 \mathrm{e}-05 \\ 7.79 \mathrm{e}+06 & 2.25 \mathrm{e}-05 \\ 6.88 \mathrm{e}+06 & 3.60 \mathrm{e}-05 \\ 6.07 \mathrm{e}+06 & 9.72 \mathrm{e}-05 \\ 4.73 \mathrm{e}+06 & 1.26 \mathrm{e}-04 \\ 3.68 \mathrm{e}+06 & 1.42 \mathrm{e}-04 \\ 2.86 \mathrm{e}+06 & 3.02 \mathrm{e}-04 \\ 2.23 \mathrm{e}+06 & 2.20 \mathrm{e}-04 \\ 1.74 \mathrm{e}+06 & 1.84 \mathrm{e}-04 \\ 1.35 \mathrm{e}+06 & 1.50 \mathrm{e}-04 \\ 1.06 \mathrm{e}+06 & 1.16 \mathrm{e}-04 \\ 8.23 \mathrm{e}+05 & 1.23 \mathrm{e}-04 \\ 6.40 \mathrm{e}+05 & 1.35 \mathrm{e}-04 \\ 5.00 \mathrm{e}+05 & 1.23 \mathrm{e}-04 \\ 3.03 \mathrm{e}+05 & 1.06 \mathrm{e}-04 \\ 1.84 \mathrm{e}+05 & 1.67 \mathrm{e}-04 \\ 6.76 \mathrm{e}+04 & 9.10 \mathrm{e}-05 \\ 2.48 \mathrm{e}+04 & 6.91 \mathrm{e}-05 \\ 9.12 \mathrm{e}+03 & 8.21 \mathrm{e}-05 \\ 3.35 \mathrm{e}+03 & 6.43 \mathrm{e}-04 \\ 1.24 \mathrm{e}+03 & 4.37 \mathrm{e}-02 \\ 4.54 \mathrm{e}+02 & 1.18 \mathrm{e}-01 \\ 1.67 \mathrm{e}+02 & 6.95 \mathrm{e}-02 \\ 6.14 \mathrm{e}+01 & 2.79 \mathrm{e}-02 \\ 2.26 \mathrm{e}+01 & 8.68 \mathrm{e}-03 \\ 8.32 \mathrm{e}+00 & 2.20 \mathrm{e}-03 \\ 3.06 \mathrm{e}+00 & 4.41 \mathrm{e}-04 \\ 1.13 \mathrm{e}+00 & 9.64 \mathrm{e}-05 \\ 4.14 \mathrm{e}-01 & 2.07 \mathrm{e}-05 \\ 1.52 \mathrm{e}-01 & 4.55 \mathrm{e}-06 \\ 1.39 \mathrm{e}-04 & \\ & \\ & \end{array}$


Table 8. Photon Source Interpolation

Calculated

$\begin{array}{ccc}\text { Group } & \begin{array}{c}\text { Energy } \\ (\mathrm{eV})\end{array} & \begin{array}{c}\text { Yield per } \\ \text { kiloton }\end{array} \\ 1 & 2.00 \mathrm{e}+07 & \\ 2 & 1.40 \mathrm{e}+07 & \\ 3 & 1.20 \mathrm{e}+07 & \\ 4 & 1.00 \mathrm{e}+07 & 8.93 \mathrm{e}-06 \\ 5 & 8.00 \mathrm{e}+06 & 5.83 \mathrm{e}-05 \\ 6 & 7.00 \mathrm{e}+06 & 2.99 \mathrm{e}-05 \\ 7 & 6.00 \mathrm{e}+06 & 7.41 \mathrm{e}-05 \\ 8 & 5.00 \mathrm{e}+06 & 3.61 \mathrm{e}-04 \\ 9 & 4.00 \mathrm{e}+06 & 4.98 \mathrm{e}-03 \\ 10 & 3.00 \mathrm{e}+06 & 3.25 \mathrm{e}-05 \\ 11 & 2.50 \mathrm{e}+06 & 6.49 \mathrm{e}-03 \\ 12 & 2.00 \mathrm{e}+06 & 8.62 \mathrm{e}-03 \\ 13 & 1.50 \mathrm{e}+06 & 9.35 \mathrm{e}-03 \\ 14 & 1.00 \mathrm{e}+06 & 7.20 \mathrm{e}-03 \\ 15 & 7.00 \mathrm{e}+05 & 8.17 \mathrm{e}-03 \\ 16 & 4.55 \mathrm{e}+05 & 5.86 \mathrm{e}-03 \\ 17 & 3.00 \mathrm{e}+05 & 5.61 \mathrm{e}-03 \\ 18 & 1.50 \mathrm{e}+05 & 1.93 \mathrm{e}-03 \\ 19 & 1.00 \mathrm{e}+05 & 5.70 \mathrm{e}-04 \\ 20 & 7.00 \mathrm{e}+04 & 3.83 \mathrm{e}-04 \\ 21 & 4.50 \mathrm{e}+04 & 8.17 \mathrm{e}-06 \\ 22 & 3.00 \mathrm{e}+04 & 5.45 \mathrm{e}-06 \\ 23 & 2.00 \mathrm{e}+04 & 1.09 \mathrm{e}-05 \\ & 1.00 \mathrm{e}+01 & \\ & & \text { sum }= \\ & & 5.97 \mathrm{e}-02\end{array}$

Literature

$\begin{array}{cc}\text { Energy } & \text { Yield per } \\ (\mathrm{eV}) & \text { kiloton }\end{array}$

$\begin{array}{ll}1.00 \mathrm{e}+07 & 9.94 \mathrm{e}-07 \\ 9.00 \mathrm{e}+06 & 7.94 \mathrm{e}-06 \\ 8.00 \mathrm{e}+06 & 5.83 \mathrm{e}-05 \\ 7.00 \mathrm{e}+06 & 2.99 \mathrm{e}-05 \\ 6.00 \mathrm{e}+06 & 7.41 \mathrm{e}-05 \\ 5.00 \mathrm{e}+06 & 3.61 \mathrm{e}-04 \\ 4.00 \mathrm{e}+06 & 4.98 \mathrm{e}-03 \\ 3.00 \mathrm{e}+06 & 3.25 \mathrm{e}-05 \\ 2.50 \mathrm{e}+06 & 6.49 \mathrm{e}-03 \\ 2.00 \mathrm{e}+06 & 8.62 \mathrm{e}-03 \\ 1.50 \mathrm{e}+06 & 9.35 \mathrm{e}-03 \\ 1.00 \mathrm{e}+06 & 4.44 \mathrm{e}-03 \\ 8.00 \mathrm{e}+05 & 5.52 \mathrm{e}-03 \\ 6.00 \mathrm{e}+05 & 3.66 \mathrm{e}-03 \\ 5.00 \mathrm{e}+05 & 3.88 \mathrm{e}-03 \\ 4.00 \mathrm{e}+05 & 3.72 \mathrm{e}-03 \\ 3.00 \mathrm{e}+05 & 3.68 \mathrm{e}-03 \\ 2.00 \mathrm{e}+05 & 3.86 \mathrm{e}-03 \\ 1.00 \mathrm{e}+05 & 9.49 \mathrm{e}-04 \\ 5.00 \mathrm{e}+04 & 2.72 \mathrm{e}-05 \\ 1.00 \mathrm{e}+01 & \\ \text { sum }= & 5.97 \mathrm{e}-02\end{array}$




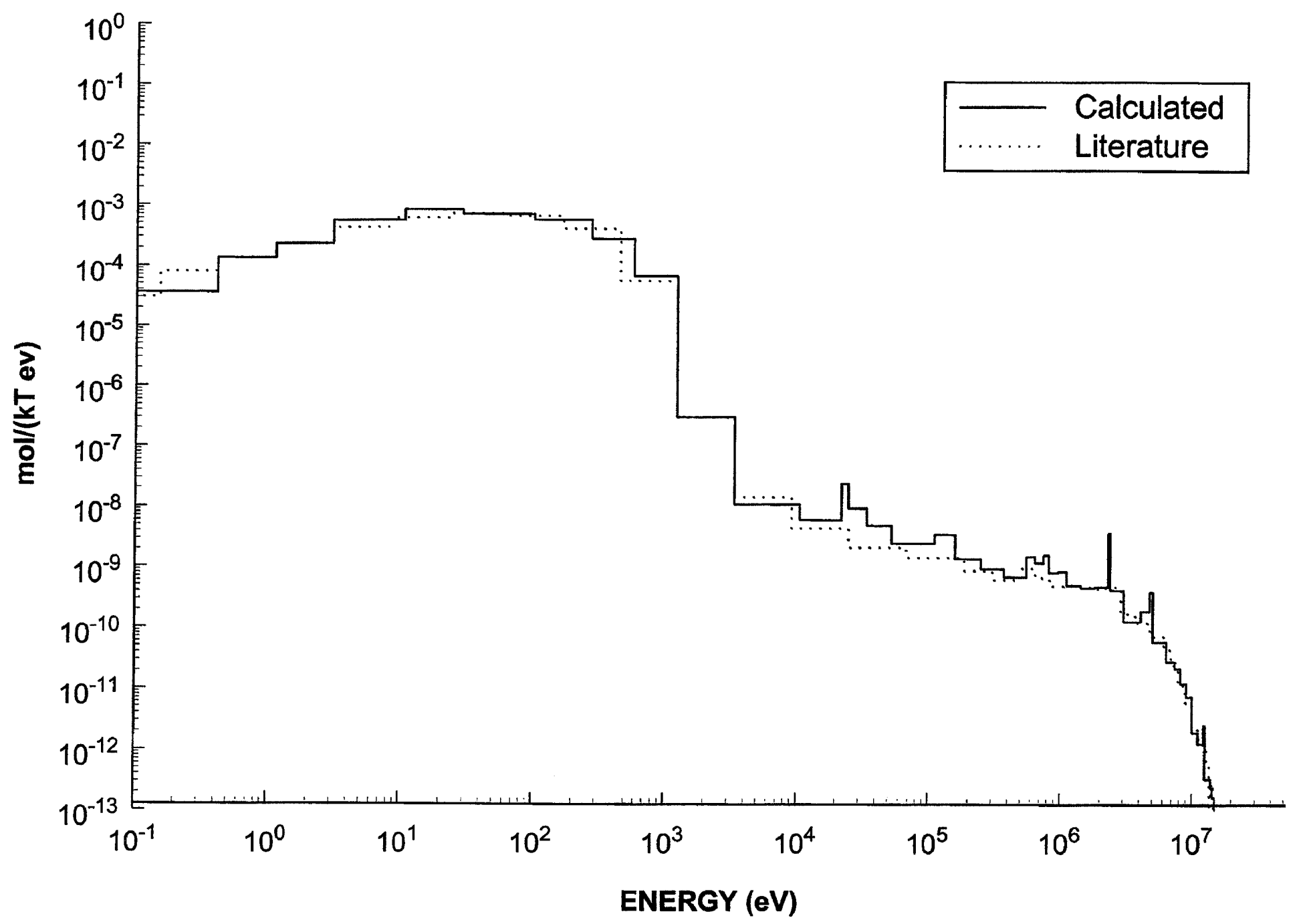

Figure 1. Neutron Source Spectra 


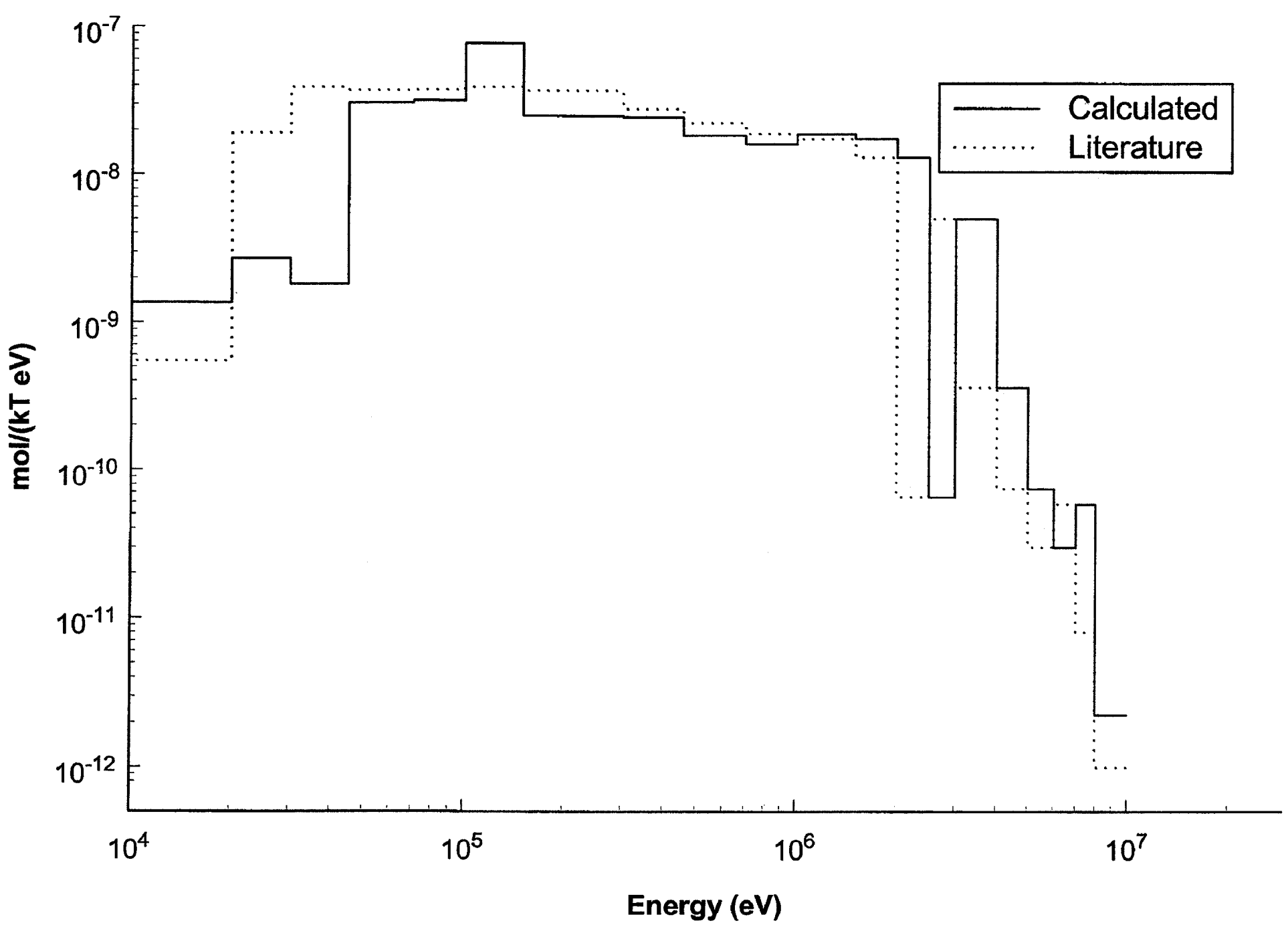

Figure 2. Photon Source Spectra 
APPENDIX A

DABL69 ABSTRACT 


\section{RSIC DATA LIBRARY DLC-130}

\section{NAME AND TITLE OF DATA LIBRARY}

DABL69: Defense Nuclear Applications Broad-Group Library based on ENDF/B-V in ANISN Format.

\section{NAME AND TITLE OF DATA RETRIEVAL PROGRAM}

BCBN: $\quad$ Convert ANISN card-image data to binary format.

\section{CONTRIBUTOR}

Oak Ridge National Laboratory, Oak Ridge, Tennessee.

\section{HISTORICAL BACKGROUND AND INFORMATION}

This data package was developed to supersede the DLC-31/FEWG1 cross section library which has been used for Defense Nuclear Agency radiation transport calculations. The availability of ENDF/B-V data coupled with some evidence that the original group structure needed some refinement, provided the driving force in the development of a replacement for FEWG1 which is based on ENDF/B-IV.

The energy group structure $(46 n, 23 g)$ is a subset of the VITAMIN-E group structure and is tailored to air-over-ground environments.

\section{APPLICATION OF THE DATA}

Based on ENDF/B-V data and a reevaluation of Fe (MOD 3) which contains significant improvements, this library consists of 80 nuclides that can be used to solve typical calculations of interest to the DNA radiation transport community. Any computer code requiring multigroup cross sections can use the data.

\section{SOURCE AND SCOPE OF DATA}

Eighty materials from the DLC-113C/VITAMIN-E library which were available on October 30,1987 are included as a first part of the DABL69 library. These materials were collapsed using the AMPX code system with the VITAMIN-E weighting functions. The second part of the DABL69 library contains 8 additional materials including $\mathrm{N}-14,0-16$, and steel using problem-dependent weighting functions generated by ANISN analysis of a typical problem. DABL69 contains 46 neutron and 23 gamma-ray energy groups. The Legendre order of expansion for angular distribution is 5.

As in DLC-31, the neutron groups are tailored to allow for the major peaks and valleys in the total neutron cross sections of nitrogen, oxygen, silicon, and iron. On the other hand, the gamma-ray groups are tailored to allow accurate calculation of pair production, annihilation photon transport, hydrogen capture, and backscatter photon transport. In the neutron energy range 0.2 to $1.8 \mathrm{MeV}$, additional boundaries were added to the parent DLC-31 group structure to give better resolution in the region where the largest fraction of the total kerma frequently occurs.

Source spectra and response functions useful in radiation transport applications are included in the library. Elemental kerma factors are provided for nuclides ranging from $\mathrm{H}-1$ to Am-243.

Version A (D00130/10360/00) includes card-image data files in both ANISN format and AMPX master format with kerma factor data derived from DLC-060/MACKLIB-IV.

Version B (D00130/10360/01) is available in ANISN card images in wich the kerma factor data has been replaced with data derived from DLC-160/KAOS-LIB-V. 


\section{DISCUSSION OF THE DATA RETRIEVAL PROGRAM}

BCBN will convert the data in ANISN format from card image to binary form. No input is required.

\section{DATA FORMAT AND COMPUTER}

Both ANISN and AMPX Card images; IBM 360/370. (D00130/10360/00)

ANISN card images; All Computers. (D00130/10360/01)

\section{TYPICAL RUNNING TIME}

Not applicable.

\section{REFERENCES}

\section{a. Included in documentation:}

"Notes on Contents of DLC-130/DABL-69" (November 22, 1993).

D.T. Ingersoll, R.W. Roussin, C.Y. Fu, and J.E. White, "DABL69: A Broad-Group Neutron/Photon Cross-Section Library for Defense Nuclear Applications," ORNL/TM-10568 (June 1989).

\section{b. Background information:}

C.R. Weisbin, R.W. Roussin, J.J. Wagschal, J.E. White, and R.Q. Wright, "Vitamin-E: An ENDF/B-V Multigroup Cross Section Library for LMFBR Core and Shield, LWR Shield, Dosimetry and Fusion Blanket Technology," ORNL-5505 (ENDF-274) (1979).

Radiation Shielding Information Center Data Package DLC-113C/VITAMIN-E, "A Coupled 174Neutron, 38-Gamma-ray Multigroup Cross-Section Library for Deriving Application-Dependent Working Libraries for Radiation Transport Calculations," contributed by Oak Ridge National Laboratory.

Radiation Shielding Information Center Data Package DLC-160/KAOS-LIB-V, "A Library of Nuclear Response Functions Generated by KAOS-V Code from ENDF/B-V and Other Data Files" (March 1991).

\section{CONTENTS OF LIBRARY}

Included is the referenced document and the cross sections and retrieval program transmitted on 1 cartridge tape in tar format or 10 DS/HD diskettes in self-extracting compressed DOS files (D00130/10360/00) or (D00130/10360/01) on two DS/HD diskettes in self-extracting compressed DOS files.

\section{DATE OF ABSTRACT}

August 1988, revised November 1988, September 1989, November 1993.

KEYWORDS: COUPLED NEUTRON-GAMMA-RAY CROSS SECTIONS; ANISN FORMAT; NEUTRON CROSS SECTIONS; DETECTOR RESPONSE; MULTIGROUP CROSS SECTIONS; KERMA FACTORS 
APPENDIX B

KAOS-LIB-V ABSTRACT 


\section{RSIC DATA LIBRARY DLC-160}

\section{NAME AND TITLE OF DATA LIBRARY}

KAOS/LIB-V: A Library of Nuclear Response Functions Generated by KAOS-V Code From ENDF/B-V and Other Data Files

\section{NAME AND TITLE OF DATA RETRIEVAL PROGRAMS}

RETRIEVE: $\quad$ An interactive program to retrieve selected responses and perform group collapsing from the VITAMIN-E 174-group values.

\section{CONTRIBUTOR}

Argonne National Laboratory, Argonne, Illinois.

\section{HISTORICAL BACKGROUND AND INFORMATION}

The microscopic cross section data bases provide the means of computing kerma factors for many materials and for incident neutron energies up to $20 \mathrm{MeV}$. The computed kerma factors from microscopic cross sections suffer from large uncertainties that cruelly manifest themselves as negative values. It is believed that improving the nuclear data files would solve the problem. However, the effort involved in doing so is massive. There is a need for improved methods in calculating nuclear heating parameters from existing data. The creation of KAOS/LIB-V is a response to this need.

\section{APPLICATION OF THE DATA}

The library is designed for fusion and fission applications.

\section{SOURCE AND SCOPE OF DATA}

The library includes neutron kerma factors, gas production, tritium breeding cross sections, and all important reaction cross sections for a large number of materials of interest in fission and fusion systems. The library was generated with the KAOS-V code (PSR-306) employing basic nuclear data from ENDF/B-V. Auxiliary nuclear data bases, such as the Japanese library JENDL-2, were used as a source of isotopic cross sections when these data are not provided in ENDF/B-V files for a natural element. KAOS/LIB-V employs the VITAMIN-E weighting function and energy group structure of 174 neutron groups.

7. DISCUSSION OF THE DATA RETRIEVAL PROGRAMS

RETRIEVE is an interactive program written in Fortran 77 to retrieve selected responses and perform group collapsing from the VITAMIN-E 174-group values.

8. DATA FORMAT AND COMPUTER

Card images; Cray computers.

\section{TYPICAL RUNNING TIME}

None noted.

10. REFERENCES

a. Included in the package: 
Y. Farawila, Y. Gohar, and C. Maynard, "KAOS/LIB-V: A Library of Nuclear Response Functions Generated by KAOS-V Code From ENDF/B-V and Other Data Files," ANL/FPP/TM-241 (April 1989).

b. Background Material:

Y. Gohar, "Kerma Factors and Activation Analyses," Paper presented at Specialists Meeting on Shielding Experiments and Analyses, Garching, Federal Republic of Germany, Feb. 12-14, 1990.

\section{CONTENTS OF PACKAGE}

The package contains the reference listed in (10.a), and the data library. The data library is transmitted on one DS/HD 5.25-inch diskette (1.2 MB).

\section{DATE OF ABSTRACT}

March 1991.

KEYWORDS: BASED ON ENDF/B-V; DAMAGE CROSS SECTIONS; KERMA FACTORS; REACTION CROSS SECTIONS 
APPENDIX C

RESPONSE FUNCTIONS IN THE KAOS/LIB-V LIBRARY 
There are 22 nuclear response function tables for each material in the library. These responses are outlined below:

1- Recommended Prompt Kerma Factor: The recommended prompt kerma factor can be either calculated by direct energy balance, kinematics, or a combination of these methods in different energy ranges according to the description in the summary provided in section III. This response is provided for all the materials included in this library. The response is given in units of (ev.barn)

2- Recommended Kerma Factor with Charged Particle Decay Heat: In this response, the decay heat deposited locally via the emitted charged particles are added to the recommended set of prompt kerma factors. This response is provided for all materials. In case there is no charged particle decay heat (within the half-life cutoff of 1 day), this response will be identical to the recommended prompt kerma.

3- Recommended Kerma Factor Plus Total Decay Heat: In this response, the total decay heat including energy carried by charged particles and gamma rays are added to the recommended prompt kerma factor response.

4- Direct Energy Balance Prompt Kerma Factor: The prompt kerma factors produced by the direct energy balance method in units of (ev.barn) are listed. Notice that in the case gamma files are not provided in ENDF/B-V, the Direct Energy Balance Prompt kerma factor includes the gamma energy as deposited locally. It is possible to find negative kerma factors in this 1ist. These can be used for comparison purposes and in special cases to account for a global energy balance in large systems. This response is presented for most but not all materials, where:zeros are entered instead. 
5- Direct Energy Balance Kerma Factor with Charged Particle Decay Heat: Charged Particle decay heat is added to the direct energy balance prompt kerma in the same way described for the recommended set. If the prompt direct energy balance was zeroed out, this response will be also zeroed out. 6- Direct energy Balance Kerma Factor Plus Total Decay Heat: The total decay heat is added to the direct energy balance kerma in the same way described for the recommended set.

7- Kinematics Prompt Kerma Factor: The prompt kerma factor produced by the kinematics method is listed. This response is provided for most, but not all materials.

8- Kinematics Kerma Factor with Charged Particle Decay Heat: The charged particle decay heat is added to the prompt kinematic kerma factor in the same way described for the recommended set. This response will be zeroed out in the case the prompt kinematic kerma factor is not provided.

9- Kinematics Kerma Plus Total Decay Heat: The total decay heat is added to the kinematics prompt kerma in the same way described for the third response. Again, this response will be zeroed out if the prompt kinematic set is not provided, and it will be identical to the prompt set if decay heat (within the 1 day half-life cutoff) is zero.

10- Prompt Gamma Energy Production: The total prompt gamma energy production is given in the units of (ev.barn). With the exception of Zirconium isotopes, where gamma files are not provided in the ENDF/B- $V$, this response is not calculated from kinematics, but rather by direct processing of ENDF/B-V files. In a few cases, interpolation and other minor corrections were made. 
11- Hydrogen-1 Production Cross Section: In this response the $\mathrm{H}-1$ gas production cross section is given in barns. The response is computed by summing up all proton-producing reaction cross sections multiplied by the respective number of emitted protons. For gas production responses, the delayed component of gas production due to radioactive decay is also added. 12- Deuterium Production cross Section: The production of $\mathrm{H}-2$ gas is presented in the same way as in response 11.

13- Tritium Production Cross Section: The production of $\mathrm{H}-3$ is presented in this response in the same way as response 11.

14- Helium-3 Production Cross Section: See response 11.

15- Helium-4 Production Cross Section: See response 11.

16- Total cross Section: The total cross section is computed from the ENDF/B-V file 2 resonance parameters (if any) and MT=1 file 3 data.

17- Elastic Scattering Cross Section: Computed from ENDF/B-V resonance file 2 (if any) and MT=2 file 3 data.

18- Total Inelastic Cross Section: This response is equivalent to the data presented in ENDF/B-V file $3 M T=4$. The $(n, n x)$ and $(n, x n)$ reactions, where $x$ represents a charged particle e.g. proton or alpha particle, are not included if they are not contained in MT=4 (and equivalently MT=51-91 series).

19- Radiative Capture Cross Section: Computed from ENDF/B-V file 2 resonance parameters (if any) and file 3 MT $=102$ data.

20- Total $(n, 2 n)$ cross Section: All non-redundant reactions producing two neutrons are added to form this response. These include sequential representation (MT=6-9), direct reaction $(M T=16),(n, 2 n \alpha)$ reaction $(M T=24)$, and $(n, 2 n 2 a)$ reaction $(M T=30)$. 
21- Total $(n, 3 n)$ Cross Section: Reactions producing 3 neutrons are added to form this response. These include $(n, 3 n)$ and $(n, 3 n a)$ reactions $(M T=17$, $M T=25)$.

22- Number of Fission Neutrons * Cross Section: In this response, the total number of fission neutrons (prompt plus delayed) is given. It is computed by multiplying the total number of neutrons produced per fission obtained from ENDF/B-V file 1 by the fission cross section. The fission cross section is computed from file 2 resonance parameters and file 3 MT=18 data. 
APPENDIX D

VITAMIN-E ABSTRACT 
RSIC DATA LIBRARY DLC-113

1. NAME AND TITLE OF DATA LIBRARY

VITAMIN-E: $174 \mathrm{n}, 38 \mathrm{~g}$ Cross-Section Library in AMPX Format.

2. NAME AND TITLE OF DATA RETRIEVAL PROGRAM

No retrieval program is provided. The PSR-117MAARS or PSR-63/AMPX code packages are suggested for full implementation of the capabilities of VITAMIN-E. JCL and sample input are provided to execute sample problems using MARS.

3. CONTRIBUTOR

Oak Ridge National Laboratory, Oak Ridge, Tennessee.

\section{HISTORICAL BACKGROUND AND INFORMATION}

The succesfful application of DLC-41/VITAMIN-C, based on ENDF/B-IV, to a variety of radiation transport problems led to the development of specifications for an ENDF/B-V based cross-section library, denoted VITAMIN-E.

\section{APPLICATION OF THE DATA}

The early phases of this new cross-section library were focused on materials for fast reactor applications and were applied to benchmark testing of ENDF/B-V. More recently, requests have been made for additional materials to be added to the basic library for fusion and weapons radiation transport applications. The library is expected to perform well for radiation transport problems where thermal upscatter is not important.

\section{SOURCE AND SCOPE OF DATA}

The energy structure of VITAMIN-E contains 174 neutron and 38 gamma-ray groups and includes the 171 neutron and 36 photon groups of VITAMIN-C as a subset. The group structure has fine detail in the energy region where cross section minima occur for important shielding materials. The 174 neutron group data were processed with MINX15; the 174 neutron, 38 photon group data were processed with LAPHNGAS (AMPX III); and the 38 gamma-ray group data with SMUG (AMPX III) from DLC99/HUGO. The ENDF/B-V special purpose dosimetry, activation, and gas production files have also been processed into the VITAMIN-E group structure using XIACS2, NITAWL, and WORM.

\section{DISCUSSION OF THE DATA RETRIEVAL PROGRAM}

PSR-117MARS can be used to translate the data into binary form and to perform a variety of useful manipulations including checking, editing, self shielding, and group collapsing.

8. DATA FORMAT AND COMPUTER

Card images; IBM 3033 D001131303301).

\section{TYPICAL RUNNING TIME}

The first part of the ZPR 6/7 infinite medium problem required approximately 55,000 I/O's and 10.5 cpu minutes on the IBM 3033. This includes the 20,000 1/O's and $9 \mathrm{cpu}$ minutes required to convert the formatted library to binary. The XSDRNPM run required only $37 \mathrm{cpu}$ seconds and $6065 \mathrm{I} / \mathrm{O}$ 's, and the GIP/ANISN run took 10,060 I/O's and 38 cpu seconds. The first part of the CTR standard blanket run 
required 58,066 I/O's and 10.15 cpu minutes. The XSDRNPM problem ran for $1.3 \mathrm{cpu}$ minutes using 5147 I/O's while the GIPIANISN run required $1.16 \mathrm{cpu}$ minutes and $9160 \mathrm{I} / \mathrm{O}$ 's.

10. REFERENCE

R. W. Roussin et al., "VITAMIN-E: A Coupled 174 Neutron, 38 Gamma-Ray Multigroup CrossSection Library for Deriving Application Dependent Working Libraries for Radiation Transport Calculations," Draft ORNL-RSIC Report (November 1987).

\section{CONTENTS OF LIBRARY}

The 174n group transport cross section data are separated into six files; the 174n, 38g group photon production dath and the $38 \mathrm{~g}$ group photon transport data are each included in separate files. Special purpose meutron dosimetry, activation, and gas production data files are also provided. JCL is included to execute sample problems using MARS. One DC6150 cartridge tape in TAR format is required to obtain the entire package.

12. DATE OF ABSTRACT

July 1984; December 1984, August 1985, November 1987.

KEYWORDS: AMPX INTERFACE FORMAT; COUPLED NEUTRON-GAMMA-RAY CROSS SECTIONS; CTR NEUTRONICS CROSS SECTIONS; CTR PROCESSED CROSSSECTION LIBRARY; GAMMA-RAY CROSS SECTIONS; GAMMA-RAY PRODUCTION DATA; MULTIGROUP CROSS SECTIONS; MULTIGROUP CROSS SECTIONS BASED ON ENDF/B; NEUTRON CROSS SECTIONS; REACTION CROSS SECTIONS 


\section{APPENDIX E}

\section{VITAMIN-B6 ABSTRACT}




\section{RSIC DATA LIBRARY DLC-184}

\section{NAME AND TITLE OF DATA LIBRARY}

VITAMIN-B6: $\quad$ A Fine-Group Cross Section Library Based on ENDF/B-VI Release 3 for Radiation Transport Applications.

2. NAME AND TITLE OF DATA RETRIEVAL PROGRAMS

AIM: $\quad$ Convert AMPX master card-image data to binary format (not included).

\section{CONTRIBUTOR}

Oak Ridge National Laboratory, Oak Ridge, Tennessee.

\section{HISTORICAL BACKGROUND AND DNFORMATION}

The successful use of DLC-41/NTTAMIN-C, which was based on ENDF/B-IV, and DLC$113 /$ VITAMIN-E, which was based on ENDF/B-V, to a variety of radiation transport problems led to the development of specifications for VITAMIN-B6.

This new multigroup cross-section library based on ENDF/B-VI Release 3 data was produced and tested for light water reactor shielding and reactor pressure vessel dosimetry applications. Significant benchmark data testing of VITAMON-B6 was an integral part of this development work to accelerate the qualification. Over 50 benchmarks were calculated using the VITAMIN-B6 library. In general, results using the new data show significant improvements relative to earlier ENDF data.

\section{APPLICATION OF THE DATA}

The successful application of VITAMIN-B6 to LWR pressure vessel fluence calculations has been demonstrated. It is expected that the range of applications will be similar to previous multigroup cross section development efforts using the VITAMTN concept (generation of fine-group, pseudo problem-independent data). In the past, the VITAMMN concept has proven to be a very effective approach for fusion reactor neutronics, LMFBR core physics analysis, radiation effects of nuclear weapons, and light water reactor shielding and dosimetry. This new fine-group, pseudo problem-independent, cross-section library contains 120 nuclides. Several dosimetry response functions and kerma factors for all 120 nuclides are also included with the library. Unlike previous VITAMIN series data libraries, VITAMINB6 contains multigroup cross sections with thermal upscattering.

\section{SOURCE AND SCOPE OF DATA}

VITAMIN-B6 is derived from ENDF/B-VI nuclear data, except for two nuclides (Sn obtained from LENDL and Zirc2 obtained from ENDF/B-IV). The responses and kerma factors were also derived primarily from ENDF/B-VI. The ENDF data were processed with the PSR-3557NJOY94 code system and converted to AMPX master library format with the SMILER module of PSR-315/AMPX77.

The actual 199 neutron group boundaries in VITAMIN-B6 were selected from 175 
groups in VITAMIN-J (a European library based on the VITAMIN-C and VITAMIN-E structures) and the 27 groups used in the SCALE shielding library, with deference given to the VITAMIN-J boundaries at higher energies when the energy values are slightly different. The thermal energy range, which contains 36 neutron groups, is defined with $5.043 \mathrm{eV}$ as the uppermost boundary.

The photon energy group structure is based on a combination of the 42 gamma-ray groups in VITAMIN-J and the 18 groups in the SCALE shielding library. The top energy group extends to $30 \mathrm{MeV}$, which allows proper representation of high energy gamma rays from neutron capture at high energies. Although the cross-section for capture at neutron energies between 20 and $30 \mathrm{MeV}$ is small, such a reaction in some materials could produce gamma rays with energies between 20 and $30 \mathrm{MeV}$ (VTTAMIN-E gamma-ray groups only went up to $20 \mathrm{MeV}$ ).

Attached tables provide information on file contents.

\section{DISCUSSION OF THE DATA RETRIEVAL PROGRAM}

Modules from PSR-352/SCAMPI, which is a subset of PSR-315/AMPX77 with some improvements from CCC-545/SCALE/4.3, were used to read the ASCII files, convert them to binary, and run test cases. The AIM program, which is included in each of these three packages, reads the formatted data and writes them as unformatted records.

\section{DATA FORMAT AND COMPUTER}

Card images in AMPX master library format; all computers (D00184/ALLCP/00).

\section{TYPICAL RUNNING TIME}

Run times are problem dependent. The test cases were run at RSICC on an IBM RS/6000 using modules from the SCAMPI package. The ctrb6 case ran in about 4 minutes. The zpr 1 case ran in about 5 minutes, and the zpr 2 case ran in about 10 minutes.

\section{REFERENCES}

The following is interim documentation for DLC-184. Formal documentation is in process and will be announced in the RSICC Newsletter upon completion.

a. included in document:

RSICC, "READ.ME" (December 1996).

J. E. White, R. Q. Wright, D. T. Ingersoll, R. W. Roussin, N. M. Greene, and R. E. MacFarlane, "VITAMIN-B6: A Fine-Group Cross Section Library Based on ENDF/B-VI for Radiation Transport Applications," from Proceedings of the International Conference on Nuclear Data for Science and Technology, Gatlinburg, Tennessee, pp. 733-736 (May 1994).

J. E. White, D.T. Ingersoll, C. O. Slater, R. W. Roussin, "BUGLE-96: A Revised Multigroup Cross Section Library for LWR Applications Based on ENDF/B-VI Release 3," (presented at the American Nuclear Society Radiation Protection \& Shielding Topical Meeting, April 21-25, 1996, Falmouth, MA) (April 1996). 
ORNL/TM-2001/16

\section{INTERNAL DISTRIBUTION}
1-2. Y. Y. Azmy
3. J. M. Barnes
4. D. T. Ingersoll
5. R. A. Lillie
6. M. A. Kuliasha
7-8. J. V. Pace, III

\author{
9-10. R. T. Santoro \\ 11-12. D. B. Simpson \\ 13. Central Research Library \\ 14. Laboratory Records - RC \\ 15. Laboratory Records (for \\ submission to OSTI)
}

\section{EXTERNAL DISTRIBUTION}

16. Dr. Robert F Christy, 1230 Arden Road, Pasadena, CA 91106

17. Dr. Harry M. Cullings, Dept. of Statistics, Radiation Effects Research Foundation, 5-2 Hijiyama Park, Minami-ku, Hiroshima-shi, 732-0815 JAPAN

18. Dr. Stephen D. Egbert, SAIC, 10260 Campus Pointe Drive, MSX2, San Diego, CA 92121-1578

19. Dr. Alexandra R. Heath, Diagnostics Applications Group Leader, Los Alamos National Laboratory, MS F663, Los Alamos, NM 87545

20. Mr. Dean C. Kaul, SAIC, 10260 Campus Pointe Drive, MSX2, San Diego, CA 921211578

21. Dr. George D. Kerr, Kerr Consulting, P.O. Box 12052, Knoxville, TN 37912-0052

22. Dr. Tore Straume, University of Utah, Radiobiology Division, 729 Arapeen Drive, Suite 2334, Salt Lake City, UT 84108

23. Dr. David G. Thomassen, Office of Biological and Environmental Research, U.S. Department of Energy, ER-72, 19901 Germantown Road, Germantown, MD 208741290

24. Dr. Joseph Weiss, Office of Health Studies, U. S. Department of Energy, EH-6/270CC, 19901 Germantown Road, Germantown, MD 20874-1290

25. Dr. Paul P. Whalen, Los Alamos National Laboratory, MS D413, Los Alamos, NM 87545

26. Dr. Robert W. Young, 946 Torrey Pine Drive, Winter Springs, FL 32708-4346 